This manuscript is now published in Frontiers in Aging Neuroscience:

Bujan, A., Sampaio, A., \& Pinal, D. (2022). Resting-state

electroencephalographic correlates of cognitive reserve: Moderating the agerelated worsening in cognitive function. Frontiers in Aging Neuroscience, 988.

https://www.frontiersin.org/articles/10.3389/fnagi.2022.854928/full 



\title{
Resting-state electroencephalographic correlates of cognitive reserve: Moderating the age-related worsening in cognitive function
}

\author{
Ana Buján ${ }^{1 *}$, Adriana Sampaio ${ }^{1}$, Diego Pinal ${ }^{1}$ \\ ${ }^{1}$ Psychological Neuroscience Laboratory (PNL), Research Center in Psychology (CIPsi), School of \\ Psychology, University of Minho, Campus de Gualtar, Portugal. \\ * Correspondence: \\ Ana Buján \\ ana.bujan@psi.uminho.pt
}

Keywords: resting-state EEG; cognitive reserve; moderation; age-related decline; cognitive function; current source density; lagged-linear connectivity

\begin{abstract}
The present exploratory study aimed to investigate the resting-state electroencephalographic (rsEEG) correlates of cognitive reserve from a lifespan perspective. To this aim, current source density (CSD) and lagged-linear connectivity (LLC) measures were assessed. We firstly explored the relationship between rsEEG measures for the different frequency bands and a socio-behavioral proxy of cognitive reserve, the Cognitive Reserve Index (CRI). Secondly, we applied moderation analyses to assess if any of the correlated rsEEG measures showed a moderating role in the relationship between age and cognitive function. Moderate negative correlations were found between the CRI and occipital CSD of delta and beta 2. Moreover, inter and intra-hemispheric LLC measures were correlated with the CRI, showing a negative association with delta and positive associations with alpha 1, beta 1 , and beta 2 . Among those correlated measures, just two rsEEG variables were significant moderators of the relationship between age and cognition: occipital delta CSD and right hemispheric beta 2 LLC between occipital and limbic regions. The effect of age on cognitive performance was stronger for higher values of both measures. Therefore, lower values of occipital delta CSD and lower beta 2 LLC between right occipital and limbic regions might protect or compensate for the effects of age on cognition. Results of this exploratory study might be helpful to allocate more preventive efforts to curb the progression of cognitive decline in adults with less CR, possibly characterized by these rsEEG parameters at a neural level. However, given the exploratory nature of the present study, more conclusive work on these rsEEG measures is needed to firmly establish their role in the cognition-age relationship, for example, verifying if these measures moderate the relationship between brain structure and cognition.
\end{abstract}




\section{INTRODUCTION}

Aging population is rapidly increasing. In Europe, people aged 65 or over represented $20.8 \%$ of the total population in 2021 (Eurostat, 2022). Although this increase in life expectancy is a priori a positive fact, age is the primary risk factor for developing a neurodegenerative disease and its associated cognitive impairment (Hou et al., 2019). In 2018, 50 million people were diagnosed with dementia worldwide (Patterson, 2018). Research into factors that can protect against cognitive impairment and its progression plays a crucial role in maintaining the quality of life and preventing dependence in older adults.

Different neuroprotective mechanisms have been proved to moderate the relationship between age and cognitive status. Indeed, for the last twenty years, much has been studied about the neural mechanisms of resilience to cognitive impairment in the aging brain, mainly in the context of neurocognitive syndromes such as Alzheimer's disease (AD). Investigation of cognitively healthy older adults is also a fruitful avenue to provide information about the neural resilience mechanisms able to curb the onset or the progression of cognitive deficits as shown by the works of the Cam-CAN group (Borgeest et al., 2020; Chan et al., 2018). However, such studies on healthy aging are still scarce (see Bartrés-Faz \& Arenaza-Urquijo, 2011 for a review). In this frame, resilience has been defined as a better-thanexpected cognitive performance relative to the degree of pathology in a given individual; so, a way to cope with the effect of pathology on cognition (Arenaza-Urquijo \& Vermuri, 2020). Resilience is a general term to refer to different neuroprotective mechanisms, including various reserve-related processes such as cognitive reserve, brain reserve, brain maintenance, or compensation (Stern et al., 2020). Among them, the most investigated entity has been cognitive reserve (CR), referring to an active process indexing the flexibility of cognitive and neural processes that helps to explain the differential vulnerability of cognitive function to age-related changes and/or to brain pathology (Barulli \& Stern, 2013; Stern et al., 2020).

Quantifying CR is a matter of constant reviews and developments as it is challenging to assess it directly. Most measures are derived from socio-behavioral indexes used as indirect proxies, such as educational or occupational attainment, intelligence, leisure activities, and other lifetime experiences (Lojo-Seoane et al., 2014; Stern, 2002). Also, composite measures including different proxies of CR have been used to indirectly measure reserve (Nucci et al., 2012). However, all these measures do not tackle the whole variability behind CR (Marqués et al., 2016). Therefore, an effort to unravel the neural correlates underlying CR has been the target of several studies. In this context, CR is proposed to be supported by more adaptable functional brain processes that constitute a more direct and objective measure of CR than the socio-behavioral indexes mentioned above (Steffener \& Stern, 2012).

In this vein, brain imaging techniques, both structural (magnetic resonance imaging - MRI) and functional (functional MRI-fMRI- and positron emission tomography - PET), have been used to investigate the potential neural correlates of $\mathrm{CR}$ along the age-related cognitive continuum. Structural MRI has been mainly employed to search for anatomical correlates of brain reserve, considered the more genetic and passive component of such reserve (i.e., brain volume, number of neurons, and synapses... see Bartrés-Faz \& Arenaza-Urquijo, 2011 for a review; Arenaza-Urquijo et al., 2013). An interesting approach from structural MRI is the development of residual measures of CR that have led to significant contributions in the field (Habeck et al., 2017; Lee et al., 2019; Reed et al., 2010; Zahodne et al., 2013, 2015). Regarding fMRI and PET, both are valuable tools for looking for functional correlates of cognitive reserve, i.e., brain networks. In general, these investigations have shown an inverse association between regional blood flow in fMRI and reserve measurements in healthy older 
adults during cognitive tasks, demonstrating increased neural efficiency in individuals with higher CR (Bartrés-Faz \& Arenaza-Urquijo, 2011).

Resting-state fMRI is particularly useful when studying older adults since it avoids demanding tasks that may be confounded by potential cognitive or motor deficits (Hausman et al., 2020). Generic resting-state networks have been proposed as a promising measure of brain flexibility and CR, mainly through the study of the functional connectivity (FC) within and between brain networks (Bastin et al., 2012; Lindbergh et al., 2019; Wook Yoo et al., 2015). Further, this is considered a more accurate approach than studying specific networks for a given task, given that activation networks may be too specific and dependent on the precise brain regions involved in the task (Stern et al., 2020). Older age has been associated with weaker FC within brain networks compared to FC patterns in younger adults but with stronger functional connections between networks (Grady et al., 2016). Arenaza-Urquijo et al. (2013) found that education, as a proxy of CR, had a positive association with the FC between brain areas such as the anterior cingulate cortex and the hippocampus as well as the inferior frontal lobe, posterior cingulate cortex, and angular gyrus. As a common result, the FC between brain networks has been found to be increased for high CR participants both in healthy adults and adults with cognitive impairment (see Anthony \& Lin, 2018 for a review).

Alternatively, neurophysiological measures through electroencephalography (EEG) and magnetoencephalography (MEG) have proven to be a promising, almost inexpensive method to study the CR neural correlates. (M)EEG is particularly useful given its relatively non-invasive nature and high temporal resolution. Especially relevant in the context of FC, the oscillatory brain activity is thought to be a key index of the coordinated activity in long-range brain networks (Moezzi et al., 2019). Hence, synchronization of EEG oscillations at the same or different frequency bands between distant brain regions is considered a mechanism promoting information flow between those regions (Siegel et al., 2012). Therefore, brain oscillations can be taken as an index of brain network organization and, then, suitable to research the neural mechanisms supporting CR.

Although the literature on the EEG correlates of CR in healthy aging is not as prolific as with other imaging techniques, several findings suggest that EEG is sensitive to the electrophysiological changes associated with various CR proxies (Fleck et al., 2019). Some studies have tried to associate different resting-state EEG and MEG measures with CR (see Sneidere et al., 2020 and Balart-Sánchez et al., 2020 for reviews). For instance, Grandy et al. (2013) found that individual alpha peak frequency was highly correlated with the general factor of intelligence but without differences between younger and older adults. Fleck et al. (2017), using a CR composite assembled with years of education and verbal intelligence scores, investigated the differences in FC as reflected by rsEEG coherence between older and younger people with high and low CR. They observed higher levels of CR were associated with greater overall brain coherence in older participants, but the opposite pattern in the younger ones. Babiloni et al. (2021, 2020b) have studied rsEEG and its association with educational attainment in three different samples: cognitively normal older adults, older adults with subjective memory complaints (SMC), and older adults with amnestic mild cognitive impairment (MCI). For older adults with SMC and negative amyloid PET, higher CR (i.e., education) was related to higher alpha rhythms in posterior areas. In those SMC participants with positive amyloid, higher CR was related to smaller posterior alpha rhythms (Babiloni et al., 2020b). Finally, comparing healthy older adults and patients with amnestic-MCI, Babiloni et al. (2021) showed that higher CR may be related to changes in rsEEG posterior alpha rhythms in healthy aging and MCI patients. They interpreted the observed results in terms of neuroprotective and compensatory mechanisms of CR. There are also some rsMEG studies that have suggested specific oscillatory MEG signatures of CR. In particular, higher CR has been shown to be related to higher gamma activity (Griffa et al., 2021; Yan \& Lin, 2020), as well as to lower 
power in the delta band and higher alpha power for the oldest-old (Griffa et al., 2021). In addition, lower CR has been associated with positive gamma asymmetry in the occipital region (Yan \& Lin, 2020).

Considering all the results provided by both imaging and (M)EEG studies, it seems that CR can be reliably identified at a neural level. Moreover, Steffener and Stern's model (2012) establishes that CR can be derived either from socio-behavioral proxies or from neural measures. However, to the best of our knowledge, no studies have investigated which specific neural processes or networks can act as correlates of CR moderating the relationship between age and cognitive function.

The current perspectives regarding research on cognitive reserve have underlined the importance of studying aging following a lifespan approach to get reliable measures that reflect individual differences in brain structure and function built over the years (Arenaza-Urquijo \& Vermuri, 2020; Stern et al., 2020). The reasoning behind it is that cognitive reserve-related variables collected at different points in the lifespan can predict cognitive function later in life (Steffener \& Stern, 2012). Ideally, this approach should be longitudinal by measuring CR and cognitive function at different ages, from early to late adulthood. This could provide a range of different cognitive profiles that can be tied to agerelated changes in cognitive reserve. So, chronological age seen from this lifespan approach can be taken as a measure of life course-related brain changes impacting cognitive outcomes. Therefore, in the present study, we pursue to identify potential indices to propose more objective and neural-based correlates of CR through the rsEEG assessment in a broad age range sample (from 18 to 82 years old).

To achieve our main aim, rsEEG variables were derived from power spectral density and connectivity measures for the main EEG frequency bands in humans (delta, theta, alpha 1, alpha 2, beta 1, and beta 2) since previous studies have found differences between high and low CR participants in some of these parameters (Babiloni et al., 2020b, 2021; Fleck et al., 2017, 2019). Further, based on the recommendations of Stern et al. (2020) for the study of CR, we implemented the following statistical plan: (1) bivariate correlation analyses between a composite socio-behavioral proxy (Cognitive Reserve Index - CRI; Nucci et al., 2012) and rsEEG variables, given that the expression of the neural variables underlying $\mathrm{CR}$ has to be associated with a socio-behavioral proxy of reserve; and, (2) moderation analyses with the rsEEG variables that correlated to the socio-behavioral proxy (i.e., CRI) as moderators of the relationship between age and cognitive status, since the brain processes or networks that underlie CR must moderate the effect of brain changes on cognition. In contrast with the recommendations by Stern et al. (2020), no structural measures of brain change were collected from the participants in the present study. However, as we stated above, we adopted a lifespan approach from early to late adulthood, considering age as a proxy of life course-related brain changes, given its role as a risk factor that impacts cognitive outcomes (https://reserveandresilience.com/framework/).

Therefore, following the conceptual research model proposed by Steffener and Stern (2012), the present study aimed to analyze resting-state EEG measures as putative neural-based correlates of cognitive reserve. In this model, cognitive reserve is operationalized by behavioral, cognitive, or neural measures that can help maintain cognitive performance by moderating its relationship with age. Due to the significant deviations from current recommendations on CR research and the paucity of studies using EEG as a measure of CR, we have conceived this study as highly exploratory, which precludes us from establishing specific hypotheses. Indeed, this study aims to generate hypotheses to be explored in future research.

\section{MATERIALS AND METHODS}




\section{Participants}

Advertisements and informative talks were used to aid in recruiting young and middle-aged adults among University of Minho staff and students, as well as of healthy older adults among users from different cultural associations and day-care centers in Portugal's North region. Data were collected from 79 volunteers, of whom 56 participants met the inclusion criteria and composed the final sample. Selected participants (age range between 18 and 82 years), thus, met the following criteria: (1) intact or well-corrected sensory function (self-reported); (2) perform independently in instrumental activities of daily living according to the Portuguese version of the Lawton and Brody scale (Lawton \& Brody, 1969; Reis et al., 2012); and, (3) absence of significant neurocognitive impairment as assessed through the Mini-mental state examination (MMSE; Folstein et al., 1975). Note that cut-off scores were adapted according to the participants' years of education as established in the Portuguese version of the MMSE (illiterate: $\leq 15$; from 1 to 11 years of formal education: $\leq 22$; more than 11 years of formal education: $\leq 27$; Santana et al., 2016). The initial screening also ensured that participants had no history of stroke, transient ischemic attacks, head injury, Parkinson's disease, or other neurological and psychiatric disorders. Participants who were taking psychoactive medications or medications for sleep promotion were excluded.

Table 1 summarizes the demographic (age, sex, and educational attainment), cognitive (MMSE score, CANTAB subtests, and CANTAB composite score), and CRI (scores for the total index and the three sub-indexes of the Cognitive Reserve Index Questionnaire, CRIq. See below for details) characteristics of the final sample. In addition, Supplementary Table 1 shows the correlations of age with the demographic and cognitive variables. Except for the CRI Education sub-index and the total CRI, all variables were significantly correlated with age. The relationship between age and cognitive status, as reflected by the five CANTAB subtests used and the calculated composite score, is illustrated in the scatterplots in Figure 1.

Table 1. Demographic and cognitive characteristics (mean (SD)/ ranges or \%) for the total sample and the three groups of age

\begin{tabular}{l|l|l|l|l}
\hline Variables & Total sample $(\mathbf{n}=\mathbf{5 6})$ & Young adults $(\mathbf{n}=\mathbf{2 5})$ & Middle-age adults $(\mathbf{n}=9)$ & Older adults $(\mathbf{n}=\mathbf{2 2})$ \\
\hline Age mean & $46.50(24.33) / 18-82$ & $21.32(4.10) / 18-35$ & $54.56(10.68) / 36-64$ & $71.82(5.82) / 65-82$ \\
\hline Sex $(\%$ female $)$ & 87.50 & 92.00 & 88.90 & 81.80 \\
\hline Education years & $11.42(5.58) / 0-24$ & $14.74(2.71) / 12-23$ & $12.56(7.02) / 4-24$ & $7.18(4.68) / 0-17$ \\
\hline MMSE score & $28.86(1.46) / 23-30$ & $29.48(0.77) / 27-30$ & $28.78(1.09) / 27-30$ & $28.18(1.87) / 23-30$ \\
\hline CANTAB RTI & $298.12(79.08) / 169-622$ & $269.06(67.75) / 169-376.5$ & $317.22(118.91) / 224-622$ & $323.32(62.60) / 224-474.5$ \\
\hline CANTAB PAL & $17.13(17.32) / 0-56$ & $4.00(8.21) / 0-40$ & $24.22(16.27) / 6-49$ & $29.14(15.17) / 14-56$ \\
\hline CANTAB SSP & $5.88(1.66) / 2-9$ & $7.08(1.26) / 5-9$ & $5.22(1.30) / 3-7$ & $4.77(1.27) / 2-6$ \\
\hline CANTAB SWM & $16.11(9.68) / 0-35$ & $10.20(9.02) / 0-28$ & $17.89(8.42) / 1-29$ & $22.09(6.70) / 13-35$ \\
\hline CANTAB MTT & $254.88(148.85) /-101-656$ & $211.50(136.32) / 32-563$ & $259.44(105.03) / 124-503$ & $302.32(178.85) /-101-656$ \\
\hline CANTAB composite & $-1.65-1.10$ & $0.55(0.36) /-1.65-0.74$ & $-0.25(0.70) /-1.61-0.07$ & $-0.52(0.55) /-1.65-1.10$ \\
\hline Total CRI & $99.5(14.11) / 78-138$ & $94.88(9.37) / 85-124$ & $107.89(15.99) / 82-126$ & $101.32(16.29) / 78-138$ \\
\hline CRI Education & $98.98(18.55) / 77-151$ & $98.72(17.61) / 80-151$ & $105.78(20.45) / 80-134$ & $96.50(19.00) / 77-140$ \\
\hline CRI Working activity & $101.13(12.24) / 81-135$ & $94.88(3.28) / 91-107$ & $109.00(15.72) / 81-128$ & $105.00(14.05) / 85-135$ \\
\hline CRI Leisure & $98.80(11.49) / 70-134$ & $94.68(4.40) / 90-109$ & $103.11(13.82) / 91-134$ & $101.73(14.61) / 70-127$ \\
\hline
\end{tabular}




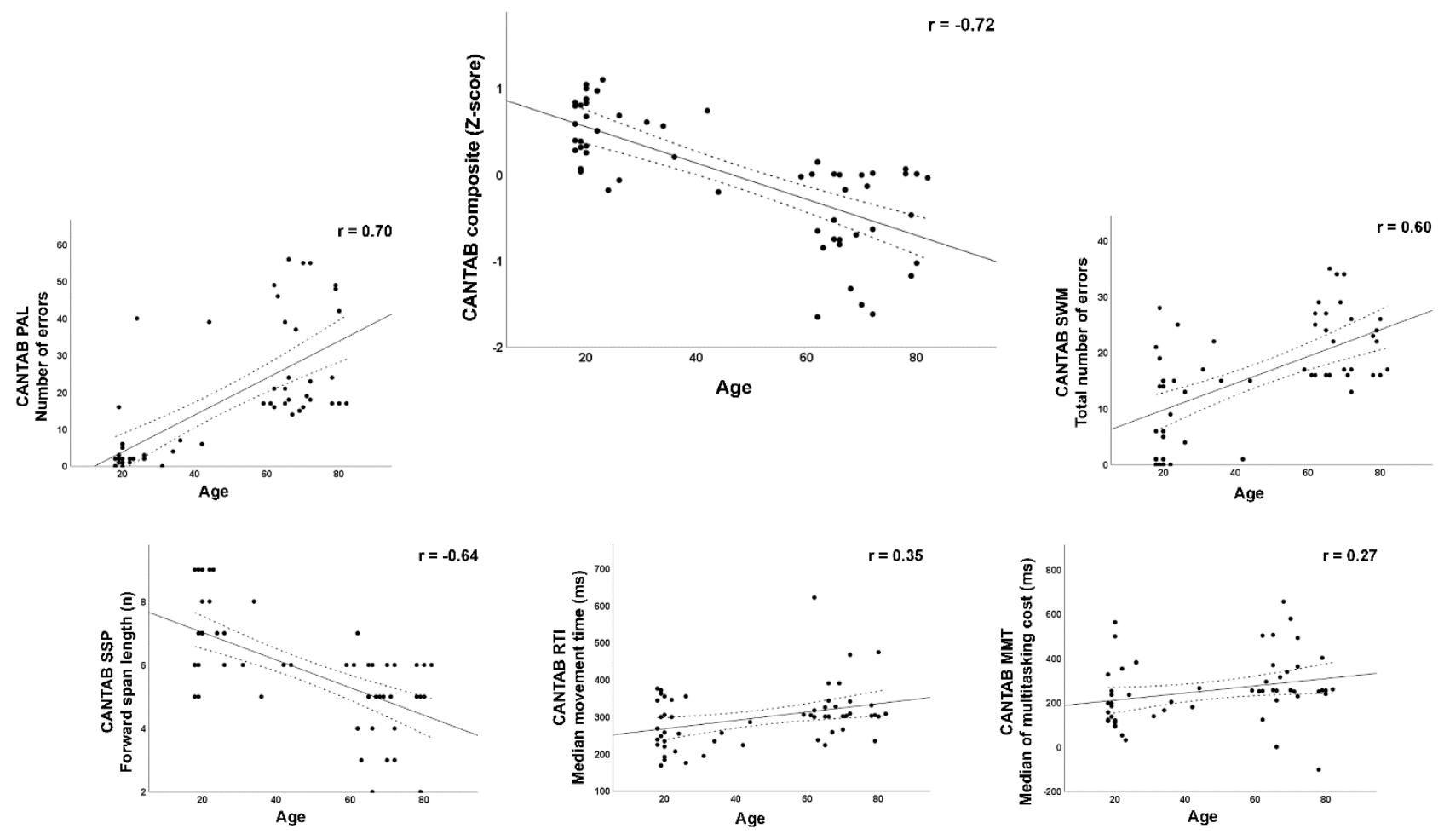

Figure 1. Scatterplots with Pearson's $r$ for the relationship between age and cognitive function measured through the CANTAB subtests and the composite score. PAL: Paired Associates Learning test; SSP: Spatial Span test; RTI: Reaction Time test; SWM: Spatial Working Memory test; MMT: Multitasking Test.

The study protocol was approved by the Institutional Review Board of the University of Minho (CE.CVS 095/2018) and conformed with the principles embodied in the Declaration of Helsinki. Before data collection, all participants were informed about the study and signed the corresponding informed consent form.

\section{Cognitive reserve and neuropsychological assessments}

The CRIq (Nucci et al., 2012) was administered to assess CR. The CRIq comprehensively estimates the amount of CR accumulated by individuals throughout their lives by collecting information related to three typical proxies of CR, i.e., school (educational level), work (type and number of years of paid work), and leisure activities (how often the individual perform different activities such as reading newspapers, performing house chores, driving, using new technologies, social activities and going to the cinema or the theater). An individual score or index is obtained for each of the three domains (CRI Education, CRI Working Activity, and CRI Leisure). Additionally, a total score (CRI) for the whole questionnaire can also be calculated and classified into one out of five categories: 1) low (CRI $\leq 70$ points); 2) medium-low (CRI between 71 and 84 points); 3) average (CRI between 85 and 114 points); 4) medium-high (CRI between 115 and 129 points); and 5) high (CRI $\geq 130$ points). 
Regarding the cognitive function assessment, several subtests of the CANTAB (Cambridge Cognition, 2019) were applied. This computerized battery has demonstrated high sensitivity to detect changes in neuropsychological performance in the aging brain, both healthy and pathological (Juncos-Rabadán et al., 2014; Junkilla et al., 2012; Marsico et al., 2014). Although there are some studies devoted to the creation of normative data for specific CANTAB tests and establishing cut-off scores for cognitive impairment in the older population (Abbot et al., 2019; De Luca et al., 2003; Robbins et al., 1994), upto-date there is no normative data for the Portuguese population. Our battery included tests to measure:

Psychomotor speed: the Reaction Time test (RTI), where the participant must hold a button at the bottom of the screen and react as soon as possible to release this bottom and select one out of five circles presented above whenever a yellow dot appears in one of them. The outcome measure in the present study was the median of the movement time.

Memory: the Paired Associates Learning test (PAL) that consists in learning the location of a series of patterns displayed behind boxes and, afterward, remembering where a specific pattern was initially located. The outcome measure used in the present study was the total number of errors made by the participant. Also, the Spatial Span test (SSP), where a sequence of squares changing in color has to be remembered in the same order as they changed, with a progressive increase in the number of squares to be remembered. The outcome measure was the forward span length (the longest sequence successfully recalled).

Executive function: the Spatial Working Memory test (SWM) for which the participant must sequentially search for yellow 'tokens' in each of several boxes. Planning and strategic thinking are needed as a box that had already contained a token cannot contain another one in the same trial. The selected outcome measure was the total number of errors (i.e., selecting boxes that have already been found to be empty and revisiting boxes that have already been found to contain a token). The Multitasking Test (MTT), where the participant has to indicate on which side of the screen an arrow appears or in which direction it is pointing according to a cue at the top of the screen (location versus direction conditions). The task has single-task blocks, when the rule is consistent across trials, as well as multitasking blocks, when the cue changes from trial to trial in a randomized order. The outcome for this test was the median of the multitasking cost; that is, the difference between the median latency of response (from stimulus appearance to button press) during multitasking blocks and that of singletask blocks.

In order to have a single measure of cognition, a CANTAB cognitive composite score was derived by computing the $\mathrm{z}$ scores for each of the five subtests and averaging across the five scores.

\section{Electroencephalographic recordings and signal pre-processing}

At least three minutes of EEG data were recorded from each participant while sitting comfortably and relaxed with eyes closed using an ActiveTwo Biosemi system (Biosemi, Amsterdam, the Netherlands) with 64 active electrodes inserted in an elastic cap according to the international 10 - 10 system. A Common Mode Sense (CMS) and Driven Right Leg (DRL) montage of two electrodes located around the vertex was used as a reference. Electrode offset was kept below $30 \mathrm{mV}$. EEG data were filtered online between $0.01-100 \mathrm{~Hz}$ and digitized at a sampling rate of $512 \mathrm{~Hz}$. Simultaneously with EEG recordings, ocular movements were recorded with two electrodes located supra and infra-orbitally to the left eye and another pair situated at the lateral canthi of each eye.

After signal storage, rsEEG data from the 64 channels were preprocessed with EEGLab (Delorme \& Makeig, 2004). First, the data were re-referenced to the nose tip, and a digital band-pass filter of $0.1-$ 
$40 \mathrm{~Hz}$ was applied. Each participant's EEG was visually inspected, and automated detection and correction of bad electrodes and bad data periods were applied using the Clean Raw Data EEGlab plugin (https://github.com/sccn/clean_rawdata). An independent component analysis algorithm was then applied to extract independent components in the remaining data. ICLabel plug-in (Pion-Tonachini et al., 2019) was used to detect residual artifacts in the independent components, which were eventually removed from the data. Afterward, the removed channels were interpolated, data were re-referenced to an average reference, and 2 seconds epochs were created. Those epochs still presenting artifacts were removed according to different criteria (abnormal values of $\pm 100 \mu \mathrm{V}$, abnormal trends with a maximum slope of $75 \mu \mathrm{V}$, presence of improbable data and abnormal distribution with a single channel limit of $5 \mathrm{SDs}$, and abnormal spectral power in frequencies from 0 to $2 \mathrm{~Hz}(50$ to $-50 \mathrm{~dB}$ ) and from 20 to $40 \mathrm{~Hz}$ ( 25 to $-100 \mathrm{~dB}$ ). Finally, as quality control, the power spectral density (PSD) was calculated through the p-welch function from the Signal Processing Toolbox, using Welch's overlapped segment averaging estimator and windowed with a Hamming window. Those recordings with a minimum of 1 minute of artifact-free data (Babiloni et al., 2020a) and standard spectra waveform in the eyes-closed resting condition were selected for posterior processing with exact low-resolution brain electromagnetic tomography (eLORETA; Pascual-Marqui et al., 2007a) to estimate the cortical sources of spectral density with a resolution of $0.5 \mathrm{~Hz}$ in six fixed bands: 1) delta $(2-4 \mathrm{~Hz})$; 2$)$ theta $(4.5-7.5$ $\mathrm{Hz})$; 3) alpha $1(8-10 \mathrm{~Hz})$; 4) alpha $2(10.5-13 \mathrm{~Hz})$; 5) beta $1(13.5-20.5 \mathrm{~Hz})$; and, 6) beta $2(21$ $30 \mathrm{~Hz})$. Despite being previously associated with CR in MEG studies, gamma activity $(30.5-40 \mathrm{~Hz})$ analyses are not included in the present work. Source estimation of gamma rhythms has been shown to perform better when using MEG as compared with EEG signals (Mideksa et al., 2015). Further, gamma rhythms are difficult to record at the scalp (Muthukumaraswamy, 2013; Nuñez \& Srinivasan, 2010), since this activity is typically highly artifacted by persistent EMG activity stemming from head and neck muscles' tension (Goncharova et al., 2003; Kropotov, 2016; Whitham et al., 2008) as well as frequent microsaccades (Yuval-Greenberg et al., 2008; Yuval-Greenberg \& Deouell, 2010) and even nasal breathing frequency (Tort et al. 2021). Nonetheless, the interested reader can find results from preliminary gamma analyses in the Supplementary Materials (Supplementary Information 1).

The average rsEEG data length used to obtain the spectral values was 2.35 mins, corresponding to a mean of 70 epochs per participant (SD: 0.39 mins / 11.64 epochs).

eLORETA is a genuine inverse solution (not merely a linear imaging method) with exact, zero error localization in the presence of measurement and structured biological noise (Pascual-Marqui, 2007a). Computations were made in a realistic head model (Fuchs et al., 2002), using the neuroanatomic Montreal Neurological Institute template (MNI152; Mazziotta et al., 2001), with the three-dimensional solution space restricted to cortical grey matter. The intracerebral volume was partitioned in 6239 voxels at $5 \mathrm{~mm}$ spatial resolution. The first processing step in eLORETA was to compute EEG crossspectra from the raw recordings using the 2-seconds epochs exported from EEGlab. Afterward, the cortical generators of surface oscillatory activity using the cross-spectra were computed. eLORETA solutions estimate current source density (CSD) values at $\mathrm{x}, \mathrm{y}$, and $\mathrm{z}$ vectors of any brain voxel able to predict EEG spectral power density at all scalp electrodes selecting the maximally smoothed solution among the possible infinite reconstructions of the active generators through a regularization procedure. This solution was normalized by the computation of the eLORETA CSD at each voxel averaged across all frequencies and all voxels. Finally, following the procedures by Babiloni et al. (2016), eLORETA solutions were averaged across all voxels in a given cortical macro-region of interest (ROI): frontal (Brodmann areas -BA-: 8, 9, 10, 11, 44, 45, 46, 47), central (BA: 1, 2, 3, 4, 6), parietal (BA: 5, 7, 30, 39, 40, 43), occipital (BA: 17, 18, 19), temporal (BA: 20, 21, 22, 37, 38, 41, 24), and limbic (BA: 31, $32,33,34,35,36)$ ROIs were considered. We estimated the current density of cortical sources as it 
provides a reference-free measurement with attenuated head volume conductor effects (Babiloni et al., 2016).

In addition, the eLORETA algorithm was also employed to obtain a measure of functional connectivity. Specifically, we calculated lagged-linear connectivity (LLC) as a measure of interdependence of rsEEG sources, given that it estimates linear inverse source connectivity while removing the artificially high zero-lag instantaneous interactions inherent to the low spatial resolution of the EEG tomography (Pascual-Marqui, 2007b). Although previous methods explore the connections between all possible pairs of locations, this "network approach" can test the joint dependence of several locations (PascualMarqui, 2007c). Hence, for each frequency band (i.e., delta, theta, alpha 1, alpha 2, beta 1, beta 2), the LLC was computed for the same six ROIs as the CSD (i.e., frontal, central, parietal, occipital, temporal, and limbic). We calculated both inter- and intra-hemispheric LLC. The inter-hemispheric LLC was calculated between all voxels of the six ROIs of each hemisphere with the corresponding ones of the other hemisphere. For the intra-hemispheric analysis, the LLC estimates were computed for all voxels of a particular ROI with all voxels of another ROI of the same hemisphere (i.e., frontal-central, frontalparietal, frontal-temporal, frontal-occipital, frontal-limbic, central-parietal, central-temporal, centraloccipital, central-limbic, parietal-temporal, parietal-occipital, parietal-limbic, temporal-occipital, temporal-limbic, and occipital-limbic for the right and the left hemispheres).

\section{Data analysis}

Before the main statistical session, an exploratory analysis of the data was carried out. There were no missing data for the demographic and health variables, the CRIq, or the EEG variables. However, for the CANTAB subtests some missing values were present (RTI, PAL and SWM $=19.6 \%$ missing; SSP and $\mathrm{MMT}=21.4 \%$ missing). These missing data were addressed by multiple imputation, creating 25 imputed datasets through a predictive mean matching procedure implemented in IBM SPSS (Version 27, IBM 2020).

In addition, given the percentage of missing data for the CANTAB subscales, to check that the multiple imputation process had worked properly, we compared the distribution of the variables from the original sample without imputations with the distribution of the same variables after the multiple imputation process. To that end, we conducted a one-way ANOVA, which pointed to the absence of significant mean differences between the two conditions for any of the CANTAB subscales (see Supplementary Information 2).

As expected, most of the rsEEG variables (i.e., eLORETA solutions) had a skewed distribution, and they were transformed to a logarithmic scale (log-10) (Babiloni et al., 2020b). No outliers ${ }^{1}$ were detected for CRIq, CANTAB, or rsEEG variables.

Descriptive and correlational analyses for demographic and neuropsychological variables were performed. Two types of inferential analyses were conducted. Firstly, the association between the proxy measure of CR and the rsEEG variables was tested through a series of bivariate correlations between CRI and each of the 252 rsEEG variables (36 CSD variables; 36 inter-hemispheric LLC variables; and 180 intra-hemispheric LLC variables). The correlation coefficients are reported as Pearson's $r$ values. Given the exploratory nature of the present study, instead of using the Bonferroni

\footnotetext{
1 Any data value was considered an outlier if it lies outside of the following ranges:

- $\quad 3$ rd quartile $+3 *$ interquartile range

- $\quad 1$ st quartile $-3 *$ interquartile range
} 
correction on associated $p$ values to assess statistical significance, we have considered for further analyses only those correlations with at least a medium effect size ( $r \geq 0.30$; Cohen et al., 1988). Additional correlational analyses between rsEEG variables and cognitive outcomes (MMSE and CANTAB measures) as well as between rsEEG variables and CRI measures are out of the scope of the present work but are publicly available at the Open Science Framework (OSF) register for this work (see below).

Secondly, to address whether rsEEG variables can be used as direct measures of CR, a moderation analysis using the regression-based approach in Hayes' (2017) PROCESS macro (Version 3.5) for IBM SPSS was conducted (Model 1; see Figure 2). Age was used as a continuous independent variable $(\mathrm{X})$, and the CANTAB composite was introduced as the dependent variable (Y). Those rsEEG variables showing moderate effect size correlations with total CRI were entered as continuous moderator variables $(\mathrm{M})$ and age $\mathrm{x}$ rsEEG variables as the interaction term $(\mathrm{X} \times \mathrm{M})$. In addition, moderation analyses were also conducted with the CRI as well as each of the three CR sub-scales of the CRIq as moderators $(\mathrm{M})$ of the relationship between age $(\mathrm{X})$ and cognitive status $(\mathrm{Y})$ to check if these CR indices are reliable proxies of CR. Moreover, additional moderation analyses were performed using each of the five CANTAB sub-scales as dependent variables (Y). Sex was entered as a covariate in all the moderation analyses. Both the independent variable (age) and the moderators (rsEEG variables and CRIq scores) were mean-centered. Regarding the multiple comparisons problem in moderation analyses, the PROCESS macro adopts a multivariate linear regression that uses a bootstrapping methodology to calculate the confidence intervals for the regression coefficients. Although this solution does not entirely rule out type I errors, it provides an efficient way to ensure that the inferences are accurate (Hayes, 2017). Therefore, bootstrapped 95\% confidence intervals (BootLLCI-BootULCI) are presented along the regression coefficients.

To probe the existence of a significant interaction, an analysis of simple slopes is included in the PROCESS macro. Thus, the conditional effect of X (age) on Y (CANTAB composite score) at relatively low $\left(16^{\text {th }}\right.$ percentile $)$, moderate $\left(50^{\text {th }}\right.$ percentile $)$, and relatively high $\left(84^{\text {th }}\right.$ percentile $)$ values of M (rsEEG measures) were evaluated through this pick-a-point procedure (Hayes, 2017). In addition, to overcome the arbitrariness of the pick-a-point approach, the Johnson-Neyman (J-N) technique also implemented in PROCESS was used in the present study to probe the significant moderation. This technique derives the values of the moderator to identify the "region of significance" of the effect of $X$ on $\mathrm{Y}$. These values of $\mathrm{M}$ demarcate the points along the continuum of $\mathrm{M}$ where the conditional effect of X on Y transitions between statistically significant and not significant levels (Hayes, 2017). 


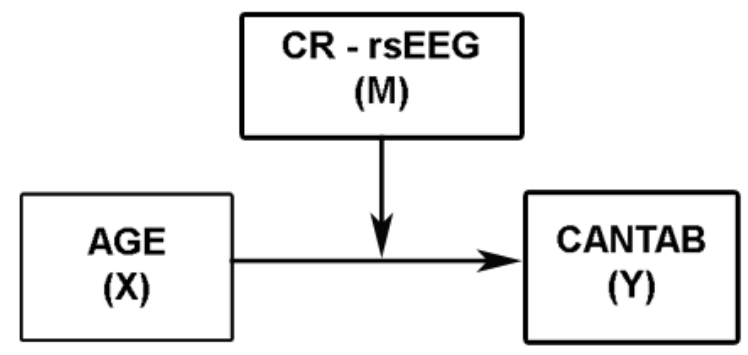

Figure 2. Conceptual representation of the simple moderation model.

\section{RESULTS}

\section{Bivariate correlations between CRI and rsEEG variables}

Moderate effect sizes were found for correlations between the CRI and several rsEEG CSD (i.e., normalized eLORETA solutions) for different frequency bands (Table 2). Negative correlations between CRI and delta and beta 2 CSD in the occipital region showed a moderate effect size (Figure $3)$.

Table 2. Pearson's correlation coefficients and 95\% confident intervals for moderate effect sizes of correlation between CRI and rsEEG variables

\begin{tabular}{ccc}
\hline rsEEG variables & Correlation Coefficient & 95\% CI \\
\hline CSD & & \\
Delta occipital & -0.36 & $-0.60,-0.08$ \\
Beta 2 occipital & -0.35 & $-0.35,-0.13$ \\
Inter-hemispherical LCC & & \\
$\quad$ Beta 1 temporal & 0.40 & $0.12,0.60$ \\
$\quad$ Beta 1 occipital & 0.35 & $0.004,0.58$ \\
Right intra-hemispherical LCC & & \\
Delta parietal-limbic & -0.44 & $-0.62,-0.22$ \\
Beta 1 frontal-occipital & 0.37 & $0.08,0.61$ \\
Beta 1 central-occipital & 0.30 & $0.02,0.51$ \\
Beta 2 occipital-limbic & 0.30 & $0.08,0.51$ \\
Left intra-hemispherical LCC & & \\
Alpha 1 frontal-temporal & 0.30 & $-0.02,0.55$ \\
Beta 1 frontal-temporal & 0.30 & $0.05,0.49$ \\
Beta 1 frontal-occipital & 0.34 & $0.04,0.55$ \\
Beta 1 parietal-temporal & 0.34 & $0.05,0.56$ \\
Beta 1 temporal-occipital & 0.35 & $0.05,0.57$ \\
\hline & & \\
\hline
\end{tabular}




\section{Delta}
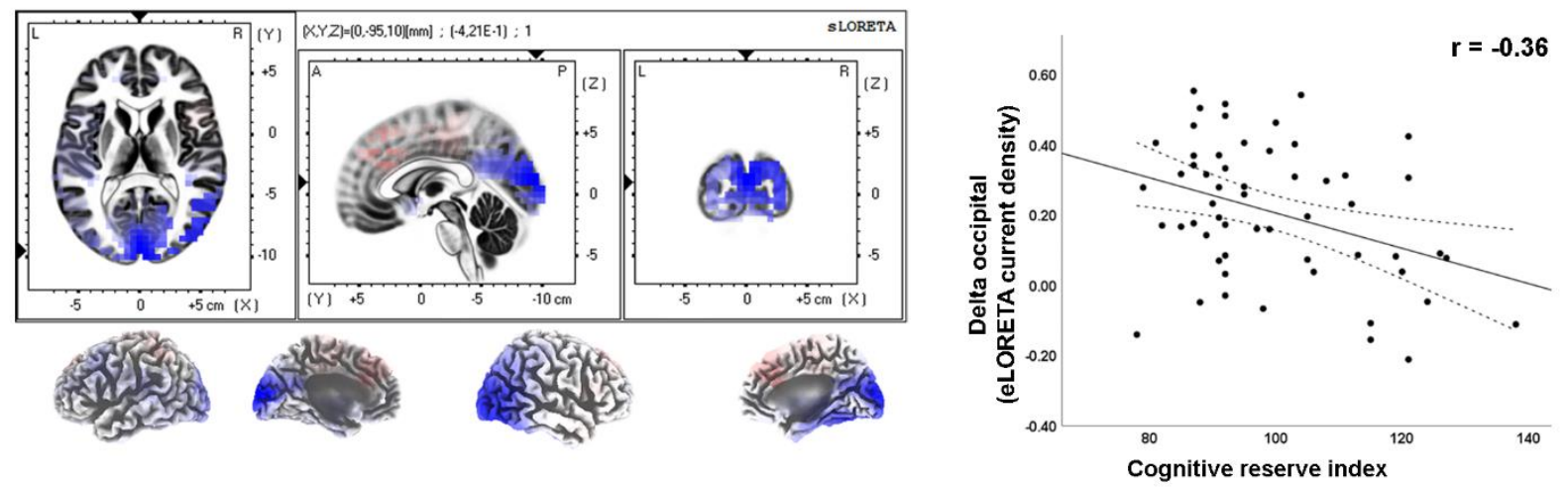

Beta 2
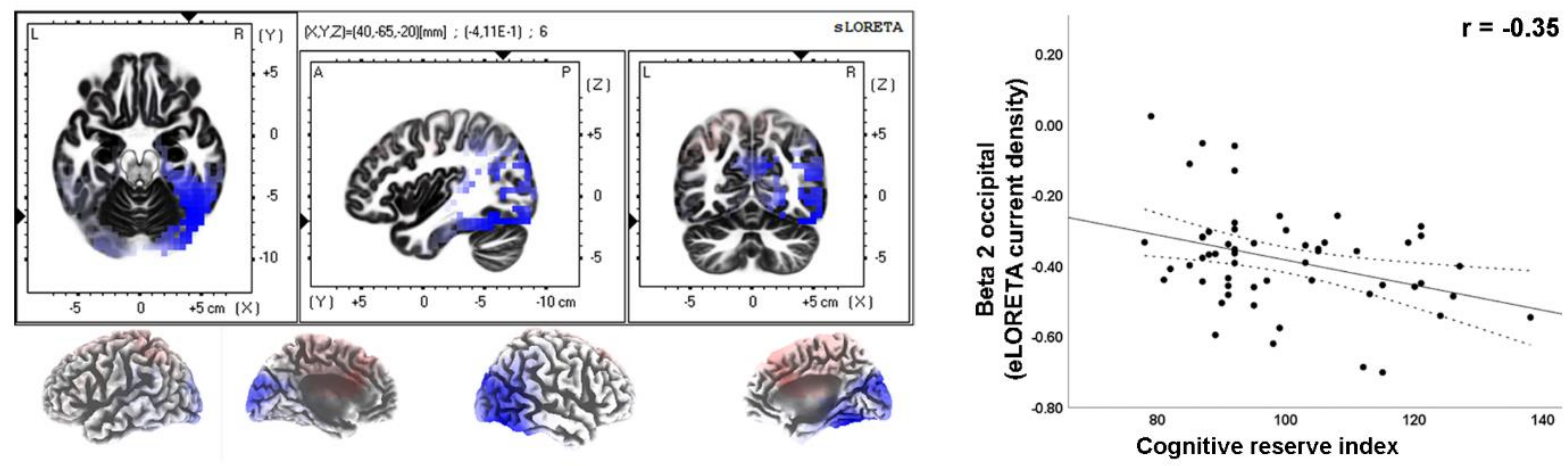

Figure 3. LORETA images and scatterplots with Pearson's $r$ for moderate correlations between CSD estimates and the Cognitive Reserve Index $(r \geq 0.30)$. The two correlations were negative (blue tones).

As regards the LLC (i.e., connectivity) measures, moderate effect sizes were found for correlations between total CRI and inter- and intra-hemispheric variables in different frequency bands (Table 2). Positive correlations were observed between CRI and beta 1 band inter-hemispheric LLC in temporal and occipital regions (Figure 4). 


\section{Beta 1}
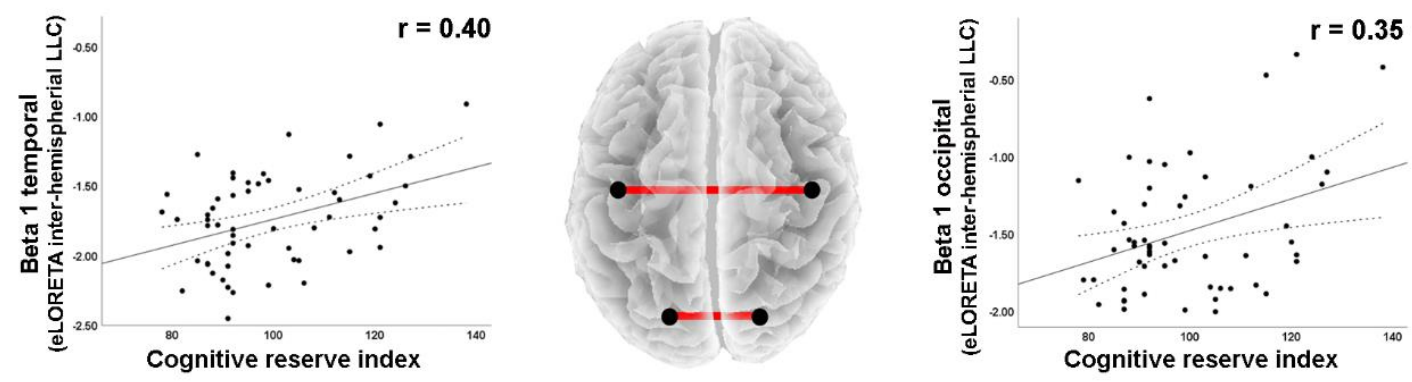

Figure 4. Graphs and scatterplots with Pearson's $r$ representing the connections (LLC measures) showing moderate size correlations between inter-hemispheric LLC estimates and the Cognitive Reserve Index ( $r \geq 0.30)$. The two correlations were positive (red lines).

A moderate negative correlation was found between CRI and delta band intra-hemispheric LLC involving parietal and limbic regions in the right hemisphere (Table 2). Positive correlations with moderate effect size were observed between CRI and intra-hemispheric connections in the right hemisphere for: (1) beta 1 band LLC between frontal and occipital, as well as between central and occipital ROIs; (2) for beta 2 LLC between occipital and limbic ROIs (Table 2; Figure 5). Regarding the left hemisphere, positive correlations with moderate effect size were observed between CRI and: (1) alpha 1 LLC between frontal and temporal; (2) beta 2 LLC between frontal and temporal, frontal and occipital, parietal and temporal, as well as temporal and occipital ROIs (Table 2; Figure 6). 

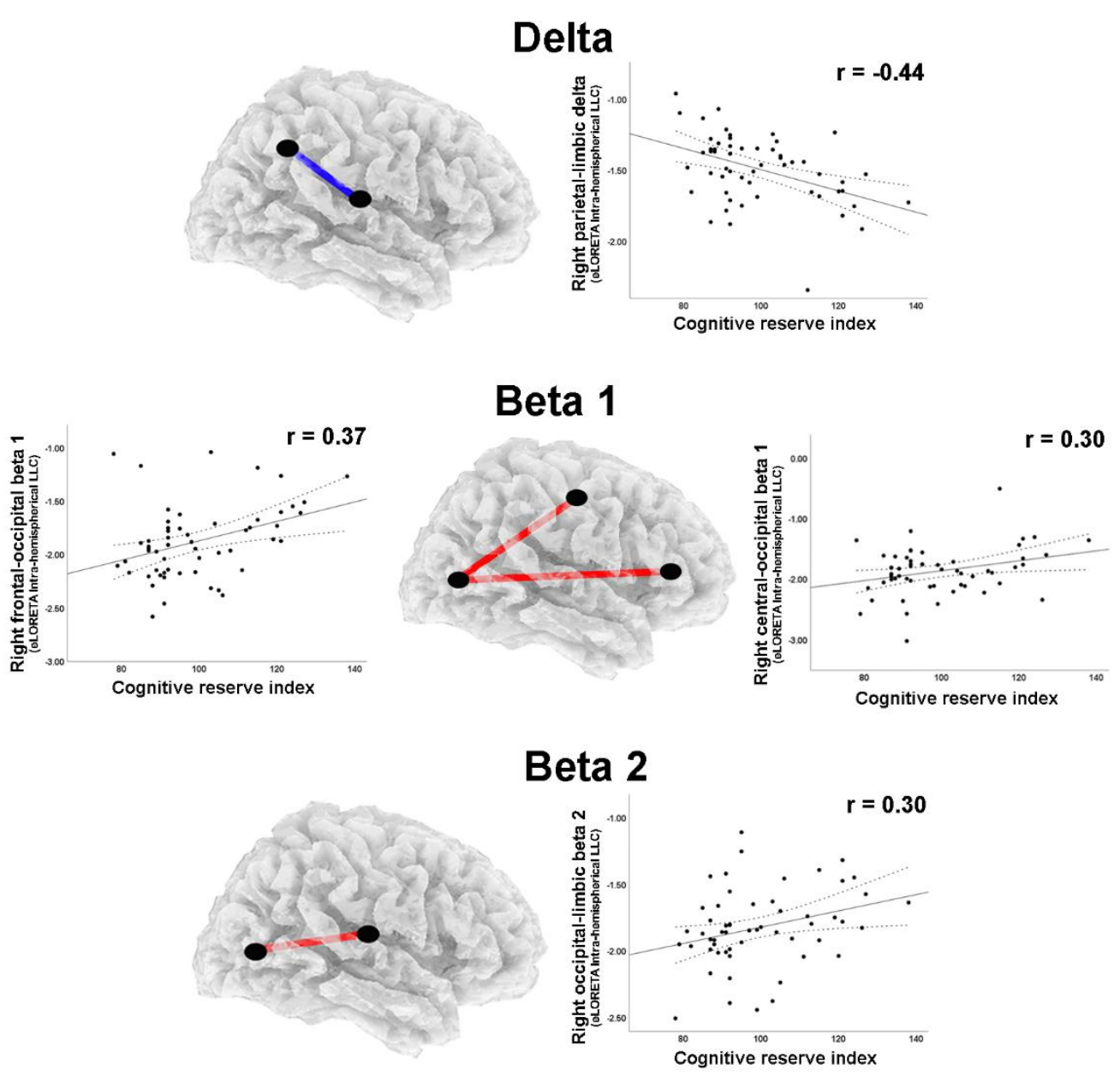

Figure 5. Graphs and scatterplots with Pearson's $r$ representing the connections (LLC measures) showing moderate size correlations between right intra-hemispheric LLC estimates and the Cognitive Reserve Index $(r \geq 0.30)$. The correlation was negative for the delta band (blue line), whereas for beta 1 and beta 2 bands, the correlations were positive (red lines). 

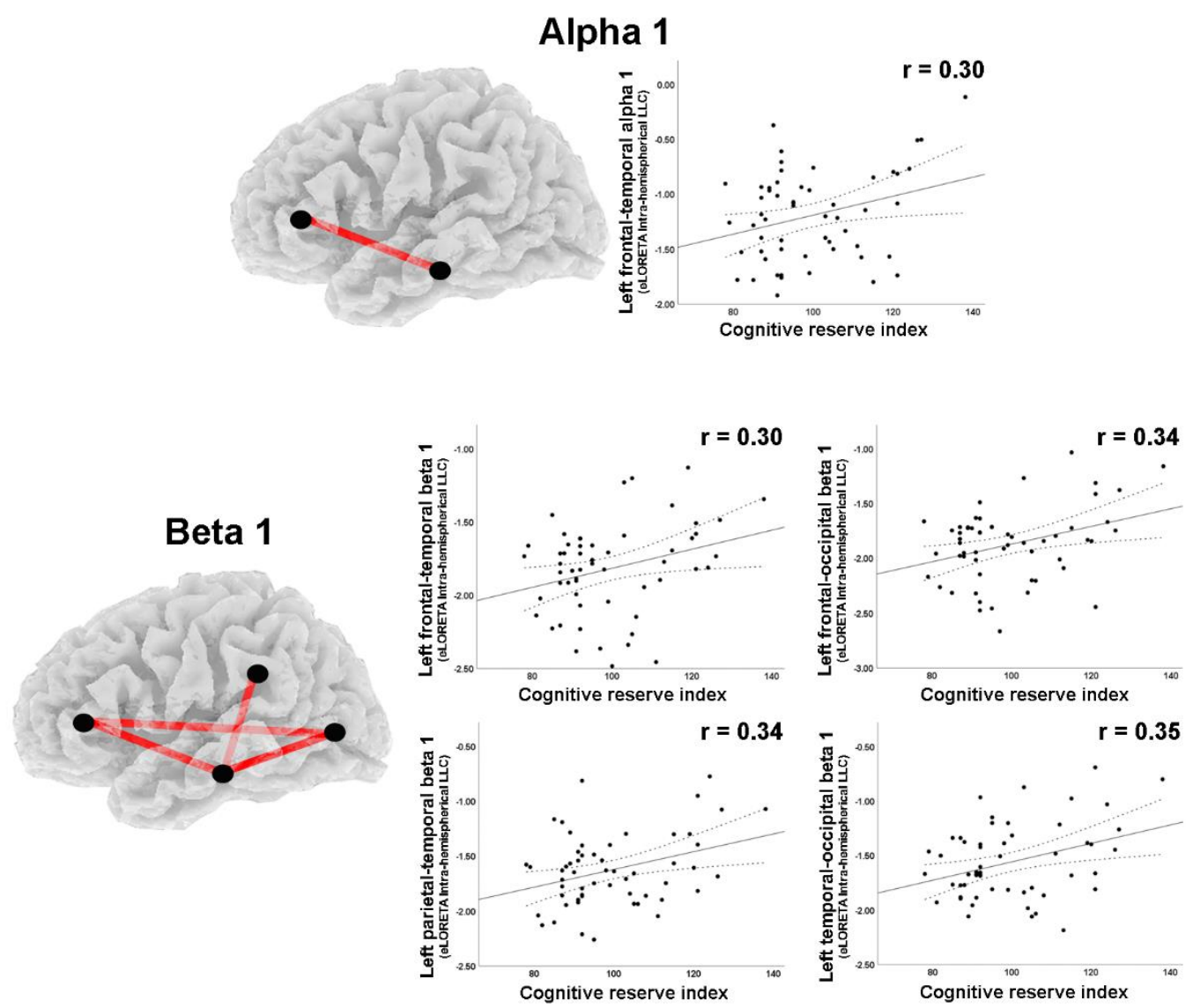

Figure 6. Graphs and scatterplots with Pearson's r representing the connections (LLC measures) showing moderate size correlations between left intra-hemispheric LLC estimates and the Cognitive Reserve Index ( $r \geq 0.30)$. The significant correlations were positive for alpha 1 and beta 1 (red lines).

\section{Moderation analyses}

For those rsEEG variables with a moderate size correlation with total CRI, moderation analyses were performed. Just two of the 13 variables significantly interacted with age to moderate cognitive status: the CSD of the delta band in the occipital ROI and the LLC of the beta 2 band in the right hemisphere between occipital and limbic regions.

For the CSD of delta activity in the occipital region, the overall regression model was statistically significant, $R^{2}=0.61, F(4,51)=19.62, p<0.001$. As expected, a negative effect of Age on CANTAB score was significant, $b=-0.02, t(51)=-8.25,95 \%$ BootLLCI $=-0.028$-BootULCI $=-0.017 ; p<0.001$, and although the effect of delta CSD was not significant, $b=-0.67, t(51)=-1.96$, BootLLCI $=-1.27$ BootULCI $=0.025 ; p=0.06$, the age $\times$ delta CSD interaction was significant, $b=-0.04, t(51)=-2.47$, BootLLCI $=-0.065$-BootULCI $=-0.009 ; p<0.05 ; \Delta R^{2}=0.05, F(1,51)=6.59$, indicating a moderator effect of delta CSD in the occipital region on the relationship between age and cognition. The covariate Sex had no effect, $b=0.19, \mathrm{t}(51)=1.04,95 \%$ BootLLCI $=-0.25$-BootULCI $=0.62 ; p=0.30$. The simple slope analysis indicated that the conditional effect of age on cognitive performance was 
statistically significant at any level of delta CSD in the occipital region (see Table 3 and Figure 7-A). However, the J-N analyses revealed that below the delta CSD value of -0.32 , the effect was not significant (Figure 7-B). Nevertheless, just 9\% of the cases (5 participants) were below that cut-off value. As shown in Figure 7, the effect of age on CANTAB score is stronger for higher levels of delta $\mathrm{CSD}$ in the occipital region. Hence, the worsening of cognitive function with age increases as the CSD of delta at the occipital region also increases.

The intra-hemispheric LLC between occipital and limbic regions for beta 2 activity in the right hemisphere was also a significant moderator of the effect of age on cognitive performance, $R^{2}=0.58$, $F(4,51)=17.83, p<0.001$. The effect of Age was significant, $b=-0.02, t(51)=-7.88$, BootLLCI $=-$ 0.03 -BootULCI $=-0.02, p<0.001$, but the effect of beta 2 intra-hemispheric LLC was not, $b=0.05$, $t(51)=0.21$, BootLLCI $=-0.41$-BootULCI $=0.53, p=0.83$. The interaction term between age and beta 2 intra-hemispheric LLC was significant, $b=-0.03, t(51)=-2.63$, BootLLCI $=-0.04$-BootULCI $=$ $0.005, p<0.05 ; \Delta R^{2}=0.06, F(1,51)=6.92$. The covariate Sex had no effect, $b=0.18, t(51)=0.94$, $95 \%$ BootLLCI $=-0.19$-BootULCI $=0.57 ; p=0.35$. Again, the conditional effect was significant for the three values of beta 2 LLC (see Table 3 and Figure 7-A). The J-N analysis established a single cutoff value for significance at -0.43 , being the lower values not significant (Figure 7-B). However, these low values were only present for $7 \%$ of the data (4 participants). As in the case of delta CSD, the effect of age on CANTAB score is stronger for higher intra-hemispheric LLC of beta 2 between occipital and limbic regions. Thus, showing an increasingly negative effect of age on cognitive performance as LLC of beta 2 between these right posterior regions increases.

Statistics for the rest of the non-significant moderation models are presented in Supplementary Table 2.

Table 3. Low $\left(16^{\text {th }}\right.$ percentile), moderate $\left(50^{\text {th }}\right.$ percentile), and relatively high $\left(84^{\text {th }}\right.$ percentile) values of the significant moderators along with statistics

\begin{tabular}{cccccccc}
\hline Moderator & M values & Effect & SE & $\boldsymbol{t}$ & $\boldsymbol{P}$ & LLCI & ULCI \\
\hline \multirow{2}{*}{ Occipital delta CSD } & -0.230 & -0.014 & 0.004 & -3.191 & 0.002 & -0.023 & -0.005 \\
& 0.005 & -0.023 & 0.003 & -8.330 & 0.000 & -0.028 & -0.017 \\
& 0.197 & -0.030 & 0.004 & -7.785 & 0.000 & 0.038 & -0.022 \\
\hline \multirow{2}{*}{$\begin{array}{c}\text { Right occipital-limbic beta 2 } \\
\text { LLC }\end{array}$} & -0.214 & -0.016 & 0.004 & -4.629 & 0.000 & -0.023 & -0.009 \\
& -0.019 & -0.021 & 0.003 & -7.688 & 0.000 & -0.027 & -0.016 \\
& 0.341 & -0.031 & 0.005 & -6.844 & 0.000 & -0.040 & -0.022 \\
\hline
\end{tabular}


A
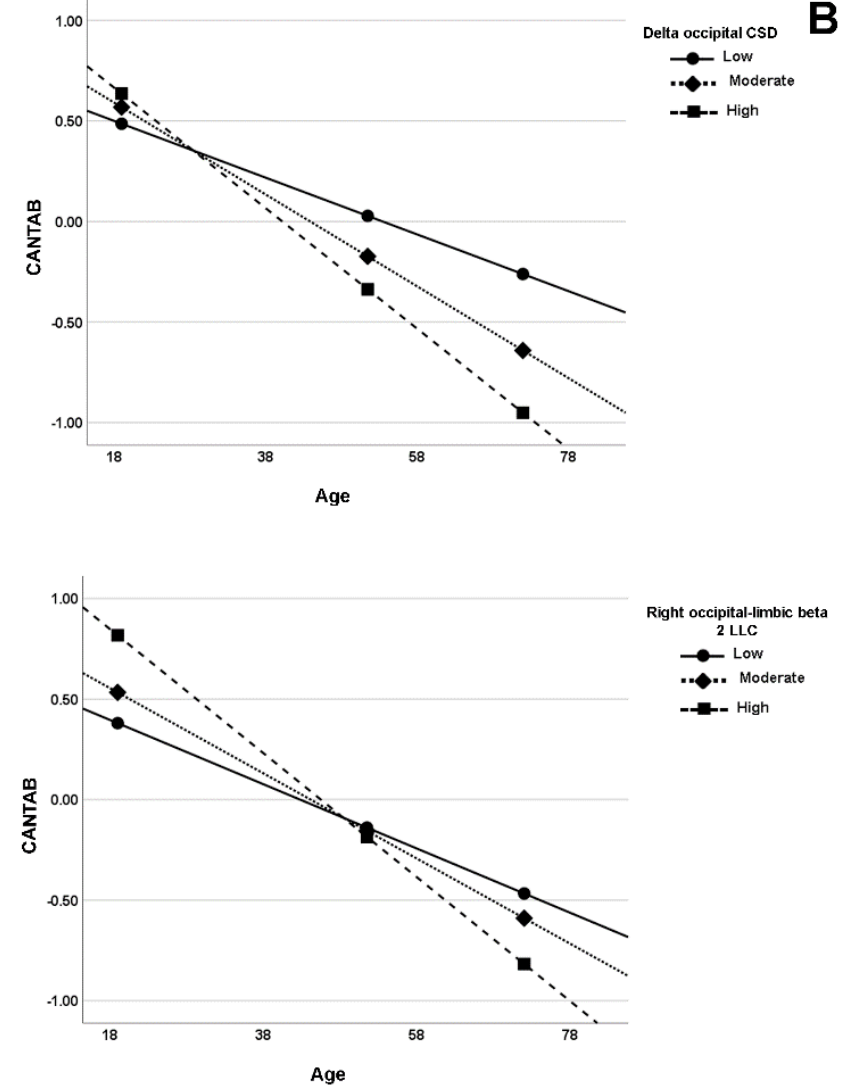

B
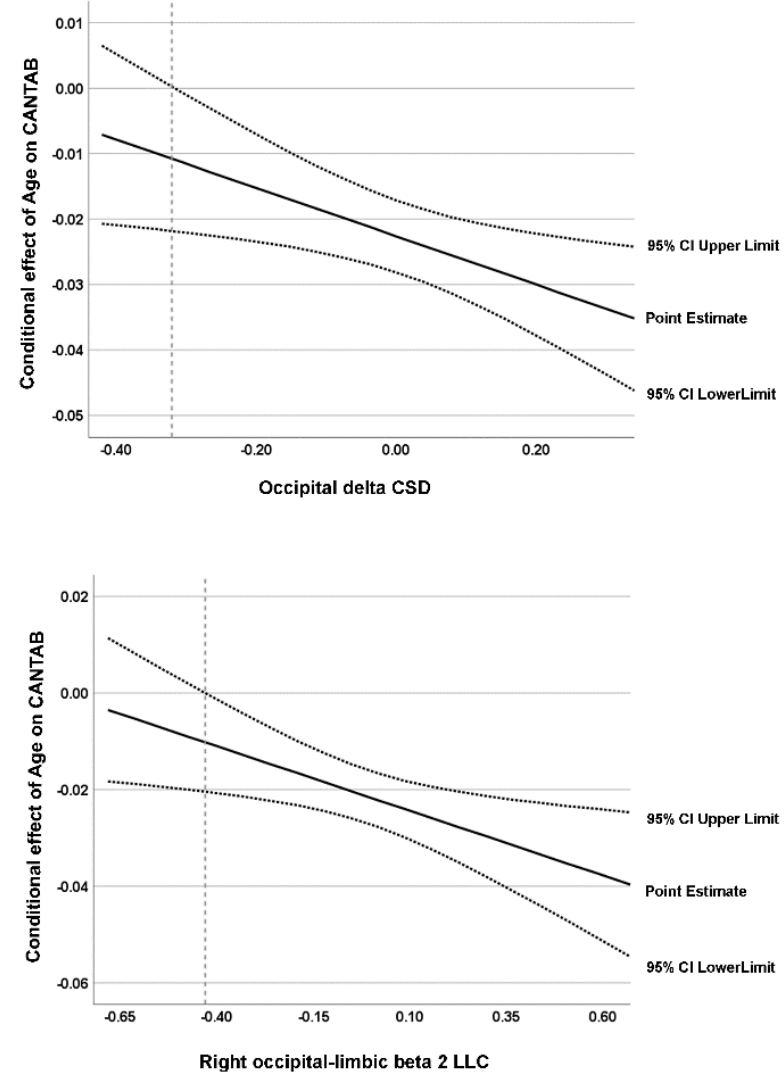

Figure 7. (A) Visual representation of the simple slopes analysis for the two significant moderators of the relationship between age and cognitive function (CANTAB composite score). Data are plotted with regression-based slopes corresponding to the conditional effect of age on cognitive function at low $\left(16^{\text {th }}\right.$ percentile), moderate $\left(50^{\text {th }}\right.$ percentile), and relatively high $\left(84^{\text {th }}\right.$ percentile) levels of estimated rsEEG measures (delta occipital CSD and right occipital-limbic beta 2 LLC). (B) Region of significance derived from the J-N technique. The region of significance is depicted as the values of the moderator corresponding to points where a conditional effect of 0 is outside of the confidence band (dotted lines). In other words, the value of the moderator for which a value of 0 is not within the confidence interval. The value from which the confidence region is outside 0 is -0.32 for occipital delta CSD and -0.43 for right occipital-limbic beta 2 LLC (dashed line). Both visual representations can be interpreted in the same way; for both measures, the higher the values, the higher the agerelated cognitive worsening.

Moderation analyses with the CRIq indices (total CRI, CRI Education, CRI Working activities, and CRI Leisure) as moderators failed to reach statistical significance for each of the possible interactions with age (see statistics data in Supplementary Table 3). In addition, we also tested the moderation analyses using the different CANTAB sub-scales as dependent variables. Significant moderations were observed in the relationship between age and SSP performance (see Supplementary Table 4.1). Thus, such association was significantly moderated by: (1) occipital delta CSD, so the higher delta activity, the higher the age-related worsening of SSP performance; (2) the inter-hemispheric LLC for temporal beta 1 activity, so the stronger the connectivity, the lower the age-related worsening of SSP 
performance; and, (3) the right intra-hemispheric LLC between frontal and occipital regions for beta 1 activity, so the stronger the connectivity, the lower the age-related worsening of SSP performance. Likewise, significant moderations were observed in the relationship between age and SWM performance (see Supplementary Table 4.2). Such association was significantly moderated by right beta 2 intra-hemispheric LLC between occipital and limbic regions, so the stronger the connectivity, the higher the age-related worsening of SWM performance. The statistics for the non-significant models can be checked in the results files in the OSF repository.

\section{DISCUSSION}

The present study aimed to analyze resting-state EEG measures (i.e., LORETA cortical sources' current density and connectivity) as possible objective neural-based correlates of cognitive reserve following the model proposed by Steffener and Stern (2012). In this conceptual research model, cognitive reserve is operationalized by behavioral, cognitive, or neural measures that can help maintain cognitive performance by moderating its relationship with age. Therefore, we analyzed the relationship between rsEEG variables, CR, age, and cognitive performance in two statistical sessions: (1) Correlational analysis, to identify those rsEEG measures with at least a moderate size association with a socio-behavioral proxy of CR, the CR index (Nucci et al., 2012); and, (2) moderation analysis, to analyze whether any of those rsEEG variables that correlate with the CRI, were also able to moderate the effect of age on cognition. The results showed that many rsEEG measures presented at least a moderate correlation, either positive or negative, with the CRI. Still, just two of them were able to moderate the relationship between age and cognitive performance. Delta CSD in the occipital region and beta 2 LLC in the right hemisphere between occipital and limbic regions are suggested as possible correlates of cognitive reserve.

\section{Correlational analysis}

Numerous neuroimaging studies have employed the correlational methodology to build potential associations between CR and structural or functional brain measures. In line with our results, in restingstate fMRI studies, higher CR has been associated with increased brain functions (i.e., stronger FC of anterior cingulate cortex with default mode network -DMN- regions, greater local efficiency, and clustering in cuneus and occipital regions), but also with decreased activity (i.e., lower metabolism in DMN and dorsal attention network regions) (see Anthony and Lin, 2018 for a review). The few rsEEG correlational studies performed up to date have shown no correlations at all (Amodio et al., 2017) or positive correlations (Grandy et al., 2013) with CR.

Despite the scarcity of rsEEG studies following the correlational approach, many studies have compared rsEEG variables between high and low CR groups. Sánchez-López et al. (2018) found less delta and theta activity in participants with high levels of physical activity, considered a protective factor for cognitive function that has been sometimes proposed as a proxy of cognitive reserve (Dik et al., 2003; Fleck et al., 2019). In addition, an increase in delta oscillatory activity has been associated with cognitive dysfunction (Babiloni et al., 2006). In this line, in the present study, an inverse correlation between the CSD of delta activity in the occipital region and the CRI was observed, so the higher the CRI, the lower the posterior delta activity. Thus, these low levels of delta might be considered as part of a neuroprotective or compensatory mechanism against cognitive decline. Further support for this assumption comes from the moderation analyses' results, as discussed below.

A negative correlation between the CRI and the CSD of beta 2 was also observed in the occipital region. In some studies, decreased brain activity in higher CR participants has been related to more efficient 
neural mechanisms, even in resting-state. For example, Bastin et al. (2012) found that a higher degree of education and verbal intelligence was associated with less metabolism of the DMN and dorsal attention network regions, concluding this may be a mechanism to optimize resting-state brain functioning. Moreover, Rogala et al. (2020) have found that resting-state beta 2 activity averaged over all electrodes negatively correlated with behavioral performance. It is likely that an increase in beta 2 activity for lower CRI values might reflect an unsuccessful compensatory mechanism reflecting higher and sustained cognitive effort in accordance with López et al. (2014). This result is probably pointing to a non-efficient organization of the functional networks. However, contrary to this tentative hypothesis, our connectivity results revealed a positive correlation between the CRI and the right intrahemispheric beta 2 LLC between occipital and limbic regions.

Inter- and intra-hemispheric connectivity are considered measures of integration of the two cerebral hemispheres and of hemispheric specialization, respectively. Further, they are paramount for good cognitive functioning (Chang et al., 2019; Liu et al., 2018). So, it seems coherent that higher CR was related to a more efficient integration and segregation between and within both hemispheres. In that sense, we observed that as the CRI increased, inter-hemispheric connectivity for beta 1 in temporal and occipital regions also increased. Similarly, intra-hemispheric LLC in the right hemisphere for beta 1 between the frontal and occipital regions, and between central and occipital regions, were also positively correlated to CRI. In the left hemisphere, alpha 1 LLC between frontal and temporal regions, and beta 1 LLC between frontal and temporal regions, and between frontal and occipital regions, as well as between the temporal region and parietal and occipital regions, also showed a positive correlation with CRI. These results are further in line with previous evidence of resting-state fMRI studies showing greater between network connectivity for high than low CR older adults (Anthony \& Lin, 2018) as well as with the higher overall brain coherence in older high reserve participants than those with lower reserve (Fleck et al., 2017). Despite in their 2017 study Fleck and coworkers found a reversed relationship between overall brain coherence and CR when comparing the younger with the older adults in their sample (age range: 45 to 64 years of age), in a posterior study taking age as a continuous variable (range age 35-75) and using LLC instead of coherence, they did not found any significant effect of age (Fleck et al., 2019). However, these authors still observed important differences in LLC between high and low CR groups, with high social CR related to greater local and long-range LLC in theta and low alpha bands, and high cognitive CR associated with greater low alpha long-range LLC between the occipital and other cortical regions. The latter results are aligned with our results, indicating positive correlations between LLC measures and CR. As Fleck and coworkers, we believe that higher connectivity may reflect a neural compensation mechanism to cope with age-related brain structure and cognition declines. Furthermore, the present results may indicate that such mechanisms are consistent across the adult lifespan, which fits within the current neurocognitive scaffolding theories of aging (Park \& Reuter-Lorenz, 2009; Reuter-Lorenz \& Park, 2014).

Nevertheless, as we noted above, increased FC is not always associated with better network functioning, both in resting-state and task-related activity (Buldú et al., 2011; Speer \& Soldan, 2015). Our results are no exception, and we observed negative correlations between CRI and intrahemispheric LLC measures for delta, namely, the weaker the LLC for delta in the right hemisphere between parietal and limbic regions, the higher the CRI. Usually, such findings are interpreted in terms of better neural efficiency and organization of brain activity in high CR individuals compared with those with lower CR. Thus, our results may, as well as those of previous studies (see, for example, Fleck et al., 2017), indicate the coexistence of compensation mechanisms, as highlighted in the previous paragraph, together with better neural efficiency and reduced need of cognitive effort as reflected in the aforementioned negative correlations. 
Alternatively, this complex pattern of positive and negative correlations between LLC and CRI may reflect differences in resting-state networks such as the DMN, dorsal attention network, and the frontoparietal network that have been previously shown to be anticorrelated in their activity (Fox et al., 2005). These resting-state functional networks have been associated with the different frequency bands studied in the present work (see Gorantla et al., 2020; Neuner et al., 2014; Hlinka et al., 2010; Mantini et al., 2007). Therefore, future studies potentially combining fMRI and EEG methods are needed to explore whether there is a dissociated pattern of relationships between those anticorrelated resting state networks and the observed EEG connectivity correlates of CR.

\section{Moderation analysis}

We performed moderation analyses with the rsEEG variables that showed a moderate correlation with CRI as moderators of the age-related prediction on cognitive scores (CANTAB composite). Out of the thirteen variables tested, just delta CSD in the occipital region and right beta 2 LLC between occipital and limbic regions were significant moderators of this relationship. More specifically, the negative relationship between age and cognitive performance observed in our sample was attenuated among people with less occipital delta activity and lower levels of right beta 2 LLC between occipital and limbic regions. As a tentative hypothesis, this might mean that $\mathrm{CR}$ could be manifested as a more efficient network with weaker activation. People with lower CR probably showed intensified activations as an attempted, although unsuccessful, compensatory mechanism.

As mentioned above, an increase in delta activity, especially in posterior areas, has been associated with cognitive impairment and reported in some pathological conditions such as AD (Babiloni et al., 2004, 2006; Benwell et al., 2020), strokes (Cassidy et al., 2020), vascular dementia (Babiloni et al., 2006; van Straaten et al., 2012), and Parkinson's disease with cognitive impairment (Caviness et al., 2011; Fonseca et al., 2013). Therefore, this mechanism is probably related to a derangement in the functional thalamo-cortical connectivity due to the neuropathology or neuroinflammatory processes (Babiloni et al., 2018). Further, resting-state delta CSD might be used as an objective index of CR since the available evidence, including our results, seems to confirm that higher delta activity could reflect cognitive vulnerability. In addition, this result is also in agreement with the results of Griffa et al. (2021) for the oldest-old participants, showing lower delta activity in a high compared to a low CR group.

A more paradoxical result was the moderation effect of the beta 2 LLC between occipital and limbic regions in the right hemisphere. The moderation analysis showed a more negative impact of age on the cognitive score as the beta 2 LLC increased, contrary to the positive correlation between occipital CSD in this frequency and CRI. This contradictory pattern seems to be due to the inclusion of the factor age and the interaction term in the moderation analyses. The effect of beta 2 LLC on CANTAB score was positive in the regression model (i.e., the higher the beta 2 LLC, the higher the CANTAB score), but when interacting with age, the effect turned negative. Although this relationship seemed the inverse at younger ages (i.e., better CANTAB scores at higher values of beta 2 LLC), as age increased, higher levels of beta 2 LLC seemed to lead to a worsened cognitive function. There are well-documented agerelated differences in rsEEG variables in the literature, even in the field of CR. For example, Fleck et al. (2017) found higher coherence during eyes-closed for older adults (i.e., adults between 59 and 65 years of age) with high CR than for those with low CR, whereas the opposite pattern was found for their younger participants (i.e., adults between 45 and 58 years of age).

A negative correlation between beta 2 activity and behavioral performance has been previously observed by Rogala et al. (2020). They consider it an electrophysiological signature of the strength of 
long-range fronto-parietal and fronto-occipital connections. In accordance with our tentative hypothesis regarding the CSD of beta 2, Rogala et al. (2020) suggested that weaker beta 2 oscillations in long-range networks might represent an enhanced capacity of network reconfiguration and, then, higher efficiency. Moreover, Santarnecchi et al. (2014) found a positive correlation between cognitive abilities and global resting-state network efficiency, characterized by weak levels of connectivity linking distant brain lobes between and within hemispheres. In addition, the functional meaning of high beta in the EEG literature has been related to anxiety and a "busy" brain (Thompson \& Thompson, 2006). Therefore, a tentative hypothesis for these lower levels of connectivity in the beta 2 band as a reflection of high levels of neural efficiency can be related to low levels of anxiety and a more regulated brain, which in turn, performs cognitively better.

Moderation analyses were also conducted using each of the five CANTAB subscales as dependent variables instead of the composite score. The obtained results were somehow confirmatory of the general results. However, the CANTAB subscales are highly correlated, so the results should be taken cautiously. In detail, the analyses for the Spatial Span test (SSP), a measure of recent memory, showed that occipital delta CSD moderated the relationship between age and SSP performance: as occipital delta CSD increases, the age-related decline for SSP performance also increases, just like the results observed for the CANTAB composite score. Besides this confirmatory result, the inter-hemispheric LLC for temporal beta 1 and the right intra-hemispheric LLC between frontal and occipital regions for beta 1 were also significant moderators of this relationship, with greater connectivity associated with a lower age-related decline in cognition. These results, again, point to the potential existence of compensatory mechanisms that are reflected in increased connectivity in high- than low-CR individuals (see Fleck et al., 2017, 2019 for similar interpretations).

In addition, for the Spatial Working Memory test (SWM), a measure of executive function, moderation results were the same as for the composite CANTAB score. Therefore, a more negative effect of age on executive function was observed as the beta 2 intra-hemispheric LLC increased. As with the present work's main result, taken together, the results of the analyses with the different CANTAB subtests may indicate the coexistence of compensatory mechanisms and increased neural efficiency processes in the resting-state brain electrical activity of high CR individuals. Nevertheless, these results suggest that performance in different cognitive domains is supported by distinct neural correlates of CR. In that vein, previous studies have shown that $\mathrm{CR}$ may be associated with specific cognitive domains while unrelated to other domains (Lavrencic, Churches \& Keage, 2017; Rodriguez, Zheng \& Chui, 2019) depending on the selected CR proxies, the cognitive tasks employed (Darby et al., 2017) or the individual experiences (Sandry, Paxton, Sumowski, 2016). Therefore, future studies are warranted to deepen the understanding of the observed neural correlates of CR and their relationship with different cognitive domains assessed through several tasks.

Finally, it is important to highlight the absence of moderation effects on the relationship between age and cognitive function by the CRI measures (total index, Education, Working activity, and Leisure sub-indices). To consider them reliable correlates of $\mathrm{CR}$, they would be expected to moderate the age and cognitive function relationship. One reason that could explain this discrepancy is that these indices may reflect the amount of CR acquired during a person's lifetime (Nucci et al., 2012) but not the CR itself. This also happens with other socio-behavioral proxies of CR, such as education. Thus, the effect of these cumulative factors would be finally translated into more efficient neural processing and brain networks, reflecting the objective pathways of CR. Consequently, as proposed by Steffener and Stern (2012), CR may be supported by more adaptable functional brain processes that constitute a more direct and objective measure of CR than socio-behavioral indexes. Further, following a lifespan approach including young adults in the sample may have obscured the moderation effect that these indices may 
have between age and cognition when studied in older populations alone, given the CR is still building up. Therefore, the rsEEG markers observed in the present study may be considered a more sensitive measure of CR-related neural changes and flexibility than paper and pencil measures such as the CRI.

In sum, many studies have proposed different neural measures as neural correlates of CR; however, as far as we know, none of them have tested the rsEEG variables as moderators in the relationship between age and cognitive status. Most of them have used the rsEEG measures as independent variables and split the sample into age and CR-based groups to perform ANOVAs. This approach presents limitations stemming from the use of an arbitrary median score to build the CR groups. Unlike previous studies, the strategy of the current work was not to compare age groups but instead to take the factor age as a continuous measure. This lifespan approach, including participants from 18 to 82 years old, with higher ages indicating possible higher physiological neural deterioration, allows us to establish which rsEEG variables may be taken as probable protective or risk markers for accelerated cognitive decline as age increases. This, in turn, justifies to some extent the absence of a structural measure of brain integrity.

Moreover, the current study followed a correlational and regressional approach, and considered CR as the ability to recruit brain networks more efficiently. In light of the reviewed evidence, CR might be reflected by increases or decreases in activity and functional connections across the brain. Indeed, current results showed that as age increases, higher levels of occipital delta CSD and stronger LLC between medial and posterior regions for beta 2 may indicate a less effective way to recruit networks in people with lower levels of CR.

Although the present results add valuable information to the research of aging and CR, they are not free from limitations and shortcomings. First, the large number of studied variables in the present study increases the probability of a type I error, and, as such, some of our results could be false positives. However, given the study's exploratory nature, which aims to generate hypotheses to be systematically and thoroughly evaluated in future studies, we adopted a rather lenient strategy regarding the problem of multiple comparisons. Second, there was a considerable amount of missing data for a dependent variable (i.e., CANTAB), which was subjected to a multiple imputation procedure. Although this is not the most desirable situation, a statistical comparison of CANTAB subtests' scores between the sample with imputed values and the sample without imputed values showed no significant differences, thus ensuring that the variable distributions were similar before and after the imputation procedure. Third, the lack of a measure of brain status did not allow us to align our results with the current research recommendations in the field of cognitive reserve (Stern et al., 2020). Hence, the study's crosssectional nature and lack of control over these brain status variables make it possible that some results were influenced by factors such as brain amyloid burden, differences in brain volume, and the presence of genetic vulnerabilities. So, the insight into CR is somewhat limited. However, we have tried to minimize this limitation by taking a lifespan perspective and considering age as a risk factor for brain changes in order to be able to suggest hypotheses and guide subsequent studies. Fourth, the sample size may not be enough to draw firm conclusions, and more middle-aged participants should be included in future studies to make the age range smoother. Another possible shortcoming of the sample is that it is primarily female, which can impose limitations on the generalizability of the presented results. It is well-known that there are differences between women and men in brain aging, and there have also been previous findings on gender differences in CR studies (see, for example, Fleck et al., 2019). However, in the present study, the variable sex was not a significant covariate in the moderation analyses. Finally, the lack of a defined and homogeneous protocol to study rsEEG and CR make the different studies not highly comparable since several methodological differences arose: the type of 
proxy measure used to obtain an estimation of CR, the different ways to calculate band amplitude or power and connectivity, the construction of the ROI(s) for the analyses, the statistical strategies, etc.

\section{CONCLUSIONS}

In the present study, we aimed to find possible neural correlates of CR that can be used as objective measures of CR instead of indirect proxies, such as educational attainment or intelligence measures. To this end, we analyzed dozens of measures of rsEEG, including eLORETA solutions for cortical current density sources and connectivity, as moderators on age-related changes in cognitive function. Moderation analyses showed that lower delta activity in the occipital region and lower connectivity of beta 2 in the right hemisphere between occipital and limbic regions could index compensatory mechanisms and increased neural efficiency to maintain high cognitive performance during the aging process. Therefore, these patterns of rsEEG activity might be considered as putative neural correlates of cognitive reserve to be deeply studied with more statistical control. As stated before, this study is highly exploratory with many variables under study, aiming to generate hypotheses to be refuted in future projects. This type of objective neural measures could be useful in the future to prevent the appearance of cognitive impairment, allocating more preventive efforts to those adults with rsEEG markers of low CR.

\section{AUTHOR CONTRIBUTIONS}

$\mathrm{AB}, \mathrm{AS}$, and DP were involved in the conception and design of the original study, as well as in the enrolment of the participants. AB and DP carried out the neuropsychological assessments and EEG recordings and performed the data analysis in terms of database construction, EEG data processing, and statistical analyses. AB, AS, and DP controlled the interpretation of the results. AB generated the first draft of the manuscript. AS and DP critically reviewed the draft and modified it accordingly. Finally, AB developed the final version of the manuscript.

\section{FUNDING}

This work was carried out at the Psychology Research Centre (CIPsi/UM- PSI/01662), which is supported by the Portuguese Foundation for Science and Technology (FCT) through the Portuguese State Budget (UIDB/01662/2020) and by the Portuguese Ministry of Education and Science through national funds and co-financed by the European Regional Development Fund (FEDER) through COMPETE2020 under the PT2020 Partnership Agreement (POCI-01-0145-FEDER-007653).

The work was directly supported by the project POCI-01-0145-FEDER-028682, PTDC/PSIGER/32152/2017 funded by FCT and the FEDER. DP was supported by the FCT grant SFRH/BPD/120111/2016. The funders had no role in study design, data collection, and analysis, decision to publish, or manuscript preparation. 


\section{ACKNOWLEDGMENTS}

We would like to thank Prof. C. Babiloni and his team for insightful comments and recommendations on the analysis approach, Dr. H. Moore, Dr. A.C. Teixeira-Santos, C. Barros, V. Ferreira, and A.R. Pereira for their assistance in data collection. We also like to thank Câmara Municipal de Vila Nova de Famalicão, Associação Gerações, Café Memoria-Braga and C.S. Vale do Homen, for their help in participants recruitment; and all those that volunteered for their participation. An online version of this study has been published as a preprint in the PsyArXiv Preprints (https://psyarxiv.com/wc82m/) 


\section{REFERENCES}

Abbott, R. A., Skirrow, C., Jokisch, M., Timmers, M., Streffer, J., van Nueten, L. et al. (2019). Normative data from linear and nonlinear quantile regression in CANTAB: Cognition in mid-tolate life in an epidemiological sample. Alzheimers Dement. (Amst). 11, 36-44. doi: 10.1016/j.dadm.2018.10.007.

Amodio, P., Montagnese, S., Spinelli, G., Schiff, S., and Mapelli, D. (2017). Cognitive reserve is a resilience factor for cognitive dysfunction in hepatic encephalopathy. Metab. Brain Dis. 32, 1287-1293. doi:10.1007/s11011-017-0032-2

Anthony, M., and Lin, F. (2018). A systematic review for functional neuroimaging studies of cognitive reserve across the cognitive aging spectrum. Arch. Clin. Neuropsychol. 33, 937-948. doi:10.1093/arclin/acx125

Arenaza-Urquijo, E. M., Landeau, B., La Joie, R., Mevel, K., Mézenge, F., Perrotin, A., et al. (2013). Relationships between years of education and gray matter volume, metabolism and functional connectivity in healthy elders. Neuroimage, $83,450-457$. doi:10.1016/j.neuroimage.2013.06.053

Arenaza-Urquijo, E. M., and Vemuri, P. (2020). Improving the resistance and resilience framework for aging and dementia studies. Alzheimers Res. Ther. 12, 1-4. doi:10.1186/s13195-020-00609-2

Babiloni, C., del Percio, C., and Buján, A. (2018). "Electroencephalography in dementing disorders" in Niedermeyer's Electroencephalography. Basic principles, clinical applications, and related fields 7 th ed, ed. D. L. Schommer, F. H. Lopes da Silva (New York: Oxford University Press), $413-432$

Babiloni, C., Barry, R. J., Başar, E., Blinowska, K. J., Cichocki, A., Drinkenburg, W. H., et al. (2020a). International Federation of Clinical Neurophysiology (IFCN)-EEG research workgroup: Recommendations on frequency and topographic analysis of resting state EEG rhythms. Part 1: Applications in clinical research studies. Clin. Neurophysiol. 131, 285-307. doi:10.1016/j.clinph.2019.06.234

Babiloni, C., Binetti, G., Cassetta, E., Dal Forno, G., Del Percio, C., Ferreri, F., et al. (2006). Sources of cortical rhythms change as a function of cognitive impairment in pathological aging: A multicenter study. Clin. Neurophysiol. 117, 252-268. doi:10.1016/j.clinph.2005.09.019

Babiloni, C., Ferri, R., Noce, G., Lizio, R., Lopez, S., Lorenzo, I., et al. (2021). Abnormalities of cortical sources of resting state alpha electroencephalographic rhythms are related to education attainment in cognitively unimpaired seniors and patients with Alzheimer's disease and amnesic mild cognitive impairment. Cereb. Cortex 31, 2220-2237. doi:10.1093/cercor/bhaa356.

Babiloni, C., Lopez, S., Del Percio, C., Noce, G., Pascarelli, M. T., Lizio, R., et al. (2020b). Restingstate posterior alpha rhythms are abnormal in subjective memory complaint seniors with preclinical Alzheimer's neuropathology and high education level: The INSIGHT-preAD study. Neurobiol. Aging 90, 43-59. doi:10.1016/j.neurobiolaging.2020.01.012.

Babiloni, C., Triggiani, A. I., Lizio, R., Cordone, S., Tattoli, G., Bevilacqua, V., et al. (2016). Classification of single normal and Alzheimer's disease individuals from cortical sources of resting state EEG rhythms. Front. Neurosci. 10 :47. doi:10.3389/fnins.2016.00047 
Babiloni, C., Binetti, G., Cassetta, E., Cerboneschi, D., Dal Forno, G., Del Percio, C., et al. (2004). Mapping distributed sources of cortical rhythms in mild Alzheimer's disease. A multicentric EEG study. Neuroimage 22, 57-67. doi:10.1016/j.neuroimage.2003.09.028

Balart-Sanchez, S. A., Bittencourt-Villalpando, M., van der Naalt, J., and Maurits, N. M. (2021). Electroencephalography, magnetoencephalography, and cognitive reserve: A systematic review. Arch. Clin. Neuropsychol. 36, 1374-1391. doi:10.1093/arclin/acaa132

Bartrés-Faz, D., and Arenaza-Urquijo, E. M. (2011). Structural and functional imaging correlates of cognitive and brain reserve hypotheses in healthy and pathological aging. Brain Topogr. 24, 340357. doi:10.1007/s10548-011-0195-9

Barulli, D., and Stern, Y. (2013). Efficiency, capacity, compensation, maintenance, plasticity: Emerging concepts in cognitive reserve. Trends Cogn. Sci. 17, 502-509. doi:10.1016/j.tics.2013.08.012

Bastin, C., Yakushev, I., Bahri, M. A., Fellgiebel, A., Eustache, F., Landeau, B., et al. (2012). Cognitive reserve impacts on inter-individual variability in resting-state cerebral metabolism in normal aging. Neuroimage 63, 713-722. doi:10.1016/j.neuroimage.2012.06.074

Benwell, C. S., Davila-Pérez, P., Fried, P. J., Jones, R. N., Travison, T. G., Santarnecchi, E., et al. (2020). EEG spectral power abnormalities and their relationship with cognitive dysfunction in patients with Alzheimer's disease and type 2 diabetes. Neurobiol. Aging 85, 83-95. doi:10.1016/j.neurobiolaging.2019.10.004

Borgeest, G. S., Henson, R. N., Shafto, M., Samu, D., Cam-CAN, and Kievit, R. A. (2020). Greater lifestyle engagement is associated with better age-adjusted cognitive abilities. Plos One 15, e0230077. doi: 10.1371/journal.pone.0230077.

Buldú, J. M., Bajo, R., Maestú, F., Castellanos, N., Leyva, I., Gil, P., et al. (2011). Reorganization of functional networks in mild cognitive impairment. PloS One 6:e19584. doi:10.1371/journal.pone.0019584

Bujan, A., Sampaio, A., \& Pinal, D. (2022, January 15). Resting-state electroencephalographic correlates of cognitive reserve: Moderating the age-related impairment in cognitive function. https://doi.org/10.31234/osf.io/wc82m

Cabeza, R., Albert, M., Belleville, S., Craik, F., Duarte, A., Grady, C. et al. (2018). Cognitive neuroscience of healthy aging: Maintenance, reserve, and compensation. Nat. Rev. Neurosci. 19, 701. doi: 10.1038/s41583-018-0068-2.

CANTAB® [Cognitive assessment software]. Cambridge Cognition (2019). All rights reserved. $\underline{\text { www.cantab.com }}$

Cassidy, J. M., Wodeyar, A., Wu, J., Kaur, K., Masuda, A. K., Srinivasan, R., and Cramer, S. C. (2020). Low-frequency oscillations are a biomarker of injury and recovery after stroke. Stroke 51, 1442-1450. doi:10.1161/STROKEAHA.120.028932 
Caviness, J. N., Lue, L., Adler, C. H., and Walker, D. G. (2011). Parkinson's disease dementia and potential therapeutic strategies. CNS Neurosci. Ther. 17, 32-44. doi:10.1111/j.17555949.2010.00216.x

Chan, D., Shafto, M., Kievit, R., Matthews, F., Spink, M., Valenzuela, M., and Henson, R. N. (2018). Lifestyle activities in mid-life contribute to cognitive reserve in late-life, independent of education, occupation, and late-life activities. Neurobiol. Aging 70, 180-183. doi: 10.1016/j.neurobiolaging.2018.06.012.

Chang, X., Collin, G., Mandl, R. C., Cahn, W., and Kahn, R. S. (2019). Interhemispheric connectivity and hemispheric specialization in schizophrenia patients and their unaffected siblings. Neuroimage Clin. 21:101656. doi:10.1016/j.nicl.2019.101656

Collaboratory on Research Definitions for Reserve and Resilience in Cognitive Aging and Dementia (2022). Framework for Terms Used in the Research of Reserve and Resilience. 38. https://reserveandresilience.com/framework/ [Accessed March 8, 2022].

Darby, R. R., Brickhouse, M., Wolk, D. A., Dickerson, B. C., \& Alzheimer's Disease Neuroimaging Initiative. (2017). Effects of cognitive reserve depend on executive and semantic demands of the task. J Neurol Neurosurg Psychiatry. 88(9), 794-802. doi:10.1136/jnnp-2017-315719

De Luca, C. R., Wood, S. J., Anderson, V., Buchanan, J. A, Proffitt, T. M., Mahony, K., et al. (2003). Normative data from the CANTAB. I: development of executive function over the lifespan. J. Clin. Exp. Neuropsychol. 25, 242-254. doi: 10.1076/jcen.25.2.242.13639.

Delorme, A., and Makeig, S. (2004). EEGLAB: An open source toolbox for analysis of single-trial EEG dynamics including independent component analysis. J. Neurosci. Methods 134, 9-21. doi:10.1016/j.jneumeth.2003.10.009

Dik, M. G., Deeg, D. J., Visser, M., and Jonker, C. (2003). Early life physical activity and cognition at old age. J. Clin.Exp. Neuropsychol. 25, 643-653. doi:10.1076/jcen.25.5.643.14583

Eurostat (2022). Population structure and ageing. https://ec.europa.eu/eurostat/statisticsexplained/index.php?title=Population_structure_and_ageing\#Past_and_future_population_agein g_trends_in_the_EU [Accessed March 15, 2022].

Fleck, J. I., Arnold, M., Dykstra, B., Casario, K., Douglas, E., and Morris, O. (2019). Distinct functional connectivity patterns are associated with social and cognitive lifestyle factors: Pathways to cognitive reserve. Front. Aging Neurosci. 11:310. doi:10.3389/fnagi.2019.00310

Fleck, J. I., Kuti, J., Mercurio, J., Mullen, S., Austin, K., and Pereira, O. (2017). The impact of age and cognitive reserve on resting-state brain connectivity. Front. Aging Neurosci. 9:392. doi:10.3389/fnagi.2017.00392

Folstein, M. F., Folstein, S. E., and McHugh, P. R. (1975). "Mini-mental state”. A practical method for grading the cognitive state of patients for the clinician. J. Psychiatr. Res. 12, 189-198. doi:10.1016/0022-3956(75)90026-6 
Fonseca, L. C., Tedrus, G. M., Carvas, P. N., and Machado, E. C. (2013). Comparison of quantitative EEG between patients with Alzheimer's disease and those with Parkinson's disease dementia. Clin. Neurophysiol. 124, 1970-1974. doi:10.1016/j.clinph.2013.05.001

Fox, M. D., Snyder, A. Z., Vincent, J. L., Corbetta, M., Van Essen, D. C., \& Raichle, M. E. (2005). The human brain is intrinsically organized into dynamic, anticorrelated functional networks. Proc Natl Acad Sci U S A. 102(27), 9673-9678. doi:10.1073/pnas.0504136102

Fuchs, M., Kastner, J., Wagner, M., Hawes, S., and Ebersole, J. S. (2002). A standardized boundary element method volume conductor model. Clin. Neurophysiol. 113, 702-712. doi:10.1016/s1388-2457(02)00030-5

Goncharova, I. I., McFarland, D. J., Vaughan, T. M., \& Wolpaw, J. R. (2003). EMG contamination of EEG: spectral and topographical characteristics. Clin. Neurophysiol., 114(9), 1580-1593. doi: 10.1016/S1388-2457(03)00093-2

Gorantla, V. R., Tedesco, S., Chandanathil, M., Maity, S., Bond, V., Lewis, C., and Millis, R. M. (2020). Associations of alpha and beta interhemispheric EEG coherences with indices of attentional control and academic performance. Behav. Neurol. 2020 doi:10.1155/2020/4672340

Grady, C., Sarraf, S., Saverino, C., and Campbell, K. (2016). Age differences in the functional interactions among the default, frontoparietal control, and dorsal attention networks. Neurobiol. Aging 41, 159-172. doi:10.1016/j.neurobiolaging.2016.02.020

Grandy, T. H., Werkle-Bergner, M., Chicherio, C., Lövdén, M., Schmiedek, F., and Lindenberger, U. (2013). Individual alpha peak frequency is related to latent factors of general cognitive abilities. Neuroimage 79, 10-18. doi:10.1016/j.neuroimage.2013.04.059

Griffa, A., Legdeur, N., Badissi, M., Van Den Heuvel, M. P., Stam, C. J., Visser, P. J., \& Hillebrand, A. (2021). Magnetoencephalography Brain Signatures Relate to Cognition and Cognitive Reserve in the Oldest-Old: The EMIF-AD 90+ Study. Front. Aging Neurosci. 13. doi: 10.3389/fnagi.2021.746373.

Habeck, C., Razlighi, Q., Gazes, Y., Barulli, D., Steffener, J., and Stern, Y. (2017). Cognitive reserve and brain maintenance: orthogonal concepts in theory and practice. Cereb. Cortex 27, 39623969. doi: 10.1093/cercor/bhw208.

Hausman, H. K., O’Shea, A., Kraft, J. N., Boutzoukas, E. M., Evangelista, N. D., Van Etten, E. J., et al. (2020). The role of resting-state network functional connectivity in cognitive aging. Front. Aging Neurosci. 12:177. doi:10.3389/fnagi.2020.00177

Hayes, A. F. (2017). Introduction to mediation, moderation, and conditional process analysis: A regression-based approach. New York: Guilford Press.

Hlinka, J., Alexakis, C., Diukova, A., Liddle, P. F., and Auer, D. P. (2010). Slow EEG pattern predicts reduced intrinsic functional connectivity in the default mode network: An inter-subject analysis. Neuroimage, 53(1), 239-246. doi:10.1016/j.neuroimage.2010.06.002 
Hou, Y., Dan, X., Babbar, M., Wei, Y., Hasselbalch, S. G., Croteau, D. L., and Bohr, V. A. (2019). Ageing as a risk factor for neurodegenerative disease. Nat. Rev. Neurol. 15, 565-581. doi: 10.1038/s41582-019-0244-7.

IBM Corp. Released 2020. (2020). IBM SPSS statistics for windows, version 27.0. [computer software]. Armonk, NY: IBM Corp.

Juncos-Rabadán, O., Pereiro, A. X., Facal, D., Reboredo, A., and Lojo-Seoane, C. (2014). Do the Cambridge Neuropsychological Test Automated Battery episodic memory measures discriminate amnestic mild cognitive impairment? Int. J. Geriatr. Psychiatry 29, 602-609. doi:10.1002/gps.4042

Junkkila, J., Oja, S., Laine, M., and Karrasch, M. (2012). Applicability of the CANTAB-PAL computerized memory test in identifying amnestic mild cognitive impairment and Alzheimer's disease. Dement. Geriatr. Cogn. Disord. 34, 83-89. doi:10.1159/000342116

Kropotov, J. (2016). Functional neuromarkers for psychiatry: Applications for diagnosis and treatment. Academic Press.

Lavrencic, L. M., Churches, O. F., \& Keage, H. A. (2018). Cognitive reserve is not associated with improved performance in all cognitive domains. Appl Neuropsychol Adult. 25(5), 473-485. doi:10.1080/23279095.2017.1329146

Lawton, M. P., and Brody, E. M. (1969). Assessment of older people: Self-maintaining and instrumental activities of daily living. Gerontologist 9, 179-186.

Lee, D. H., Lee, P., Seo, S. W., Roh, J. H., Oh, M., Oh, J. S., et al. (2019). Neural substrates of cognitive reserve in Alzheimer's disease spectrum and normal aging. NeuroImage 186, 690-702. doi: 10.1016/j.neuroimage.2018.11.053.

Lindbergh, C. A., Zhao, Y., Lv, J., Mewborn, C. M., Puente, A. N., Terry, D. P. et al. (2019). Intelligence moderates the relationship between age and inter-connectivity of resting state networks in older adults. Neurobiol. Aging 78, 121-129. doi: 10.1016/j.neurobiolaging.2019.02.014.

Liu, Y., Guo, W., Zhang, Y., Lv, L., Hu, F., Wu, R., and Zhao, J. (2018). Decreased resting-state interhemispheric functional connectivity correlated with neurocognitive deficits in drug-naive first-episode adolescent-onset schizophrenia. Int. J. Neuropsychopharmacol. 21, 33-41. doi:10.1093/ijnp/pyx095

Lojo-Seoane, C., Facal, D., Guàrdia-Olmos, J., and Juncos-Rabadán, O. (2014). Structural model for estimating the influence of cognitive reserve on cognitive performance in adults with subjective memory complaints. Arch. Clin. Neuropsychol. 29, 245-255. doi:10.1093/arclin/acu007

López, M. E., Aurtenetxe, S., Pereda, E., Cuesta, P., Castellanos, N. P., Bruña, R., et al. (2014). Cognitive reserve is associated with the functional organization of the brain in healthy aging: a MEG study. Front. Aging Neurosci. 6, 125. doi: 10.3389/fnagi.2014.00125. 
Mantini, D., Perrucci, M. G., Del Gratta, C., Romani, G. L., and Corbetta, M. (2007).

Electrophysiological signatures of resting state networks in the human brain. Proc. Natl. Acad.

Sci. U S A 104, 13170-13175. doi:10.1073/pnas.0700668104

Marques, P., Moreira, P., Magalhães, R., Costa, P., Santos, N., Zihl, J., et al. (2016). The functional connectome of cognitive reserve. Hum. Brain Mapp. 37, 3310-3322. doi:10.1002/hbm.23242

Marsico, M., de Jager, C. A., Grant, A., Zhu, X., Markwick, A., and Chandler, J. (2014). Cognitive and functional profiles in mild-to-moderate Alzheimer's disease and mild cognitive impairment compared to healthy elderly. Adv. Alzheimer Dis. 3:168. doi:10.4236/aad.2014.34016

Mazziotta, J., Toga, A., Evans, A., Fox, P., Lancaster, J., Zilles, K., et al. (2001). A probabilistic atlas and reference system for the human brain: International consortium for brain mapping (ICBM). Philos. Trans. R. Soc. Lond. B. Biol. Sci. 356, 1293-1322. doi:10.1098/rstb.2001.0915

Mideksa, K. G., Hoogenboom, N., Hellriegel, H., Krause, H., Schnitzler, A., Deuschl, G., et al. (2015). Comparison of EEG and MEG in source localization of induced human gamma-band oscillations during visual stimulus. 37th Annual International Conference of the IEEE Engineering in Medicine and Biology Society (EMBC), 8119-8122.

Moezzi, B., Pratti, L. M., Hordacre, B., Graetz, L., Berryman, C., Lavrencic, L. M., et al. (2019). Characterization of young and old adult brains: An EEG functional connectivity analysis. Neuroscience 422, 230-239. doi:10.1016/j.neuroscience.2019.08.038

Muthukumaraswamy, S. D. (2013). High-frequency brain activity and muscle artifacts in MEG/EEG: a review and recommendations. Front. Hum. Neurosci., 7, 138. doi: 10.3389/fnhum.2013.00138

Neuner, I., Arrubla, J., Werner, C. J., Hitz, K., Boers, F., Kawohl, W., et al. (2014). The default mode network and EEG regional spectral power: A simultaneous fMRI-EEG study. PLoS One 9, e88214. doi:10.1371/journal.pone.0088214

Nucci, M., Mapelli, D., and Mondini, S. (2012). Cognitive reserve index questionnaire (CRIq): A new instrument for measuring cognitive reserve. Aging Clin. Exp. Res. 24, 218-226. doi: $10.3275 / 7800$

Nunez, P. L., \& Srinivasan, R. (2010). Scale and frequency chauvinism in brain dynamics: too much emphasis on gamma band oscillations. Brain Struct Funct, 215(2), 67-71. doi: 0.1007/s00429010-0277-6

Park, D. C., \& Reuter-Lorenz, P. (2009). The adaptive brain: Aging and neurocognitive scaffolding. Annu Rev Psychol. 60, 173. doi:10.1146/annurev.psych.59.103006.093656

Pascual-Marqui, R. D. (2007a). Discrete, 3D distributed, linear imaging methods of electric neuronal activity. Part 1: Exact, zero error localization. arXiv: 0710.3341 [math-ph]. Available online at: http://arxiv.org/pdf/0710.3341 (Accessed on July 5, 2021).

Pascual-Marqui, R. D. (2007b). Coherence and phase synchronization: Generalization to pairs of multivariate time series, and removal of zero-lag contributions. arXiv: 0706.1776v3 [stat.ME]. Available online at: http://arxiv.org/pdf/0706.1776 (Accessed on July 5, 2021). 
Pascual-Marqui, R. D. (2007c). Instantaneous and lagged measurements of linear and nonlinear dependence between groups of multivariate time series: Frequency decomposition. arXiv: 0711.1455 [stat.ME]. Available online at: http://arxiv.org/abs/0711.1455 (Accessed on July 5, 2021).

Patterson, C. (2018). World Alzheimer report 2018: The state of the art of dementia research: New frontiers. London, UK: Alzheimer's Disease International (ADI).

Pion-Tonachini, L., Kreutz-Delgado, K., and Makeig, S. (2019). ICLabel: An automated electroencephalographic independent component classifier, dataset, and website. Neuroimage 198, 181-197. doi:10.1016/j.neuroimage.2019.05.026

Reed, B. R., Mungas, D., Farias, S. T., Harvey, D., Beckett, L., Widaman, K., et al. (2010). Measuring cognitive reserve based on the decomposition of episodic memory variance. Brain 133, 2196-2209. doi: 10.1093/brain/awq154.

Reis, G., Teixeira, L., Constança, P., and Martins, M. (2012). Independencia en las actividades de la vida: Estudio de validación de una escala para la población portuguesa [Independence in the performance of daily life activities (ADLs): Validating a research scale applied to the portuguese population]. Gerokomos 23(2), 69-73.

Reuter-Lorenz, P. A., \& Park, D. C. (2014). How does it STAC up? revisiting the scaffolding theory of aging and cognition. Neuropsychol Review. 24(3), 355-370. doi:10.1007/s11065-014-9270-9

Robbins, T. W., James, M., Owen, A. M., Sahakian, B. J., McInnes, L., and Rabbitt, P. (1994). Neuropsychological Test Automated Battery (CANTAB): a factor analytic study of a large sample of normal elderly volunteers. Dementia 5, 266-281. doi: 10.1159/000106735.

Rodriguez, F. S., Zheng, L., Chui, H. C., \& Aging Brain: Vasculature, Ischemia, and Behavior Study. (2019). Psychometric characteristics of cognitive reserve: How high education might improve certain cognitive abilities in aging. Dement Geriatr Cogn Disord. 47(4-6), 335-344. doi:10.1159/000501150

Rogala, J., Kublik, E., Krauz, R., and Wróbel, A. (2020). Resting-state EEG activity predicts frontoparietal network reconfiguration and improved attentional performance. Sci. Rep. 10, 1-15. doi:10.1038/s41598-020-61866-7

Sanchez-Lopez, J., Silva-Pereyra, J., Fernández, T., Alatorre-Cruz, G. C., Castro-Chavira, S. A., González-López, M., and Sánchez-Moguel, S. M. (2018). High levels of incidental physical activity are positively associated with cognition and EEG activity in aging. PLoS One 13, e0191561. doi:10.1371/journal.pone.0191561

Sandry, J., Paxton, J., \& Sumowski, J. F. (2016). General mathematical ability predicts PASAT performance in MS patients: Implications for clinical interpretation and cognitive reserve. J Int Neuropsychol Soc. 22(3), 375-378. doi:10.1017/S1355617715001307

Santana, I., Duro, D., Lemos, R., Costa, V., Pereira, M., Simões, M. R., and Freitas, S. (2016). Minimental state examination: Screening and diagnosis of cognitive decline, using new normative data. Acta Med. Port. 29, 240-248. doi:10.20344/amp.6889 
Santarnecchi, E., Galli, G., Polizzotto, N. R., Rossi, A., and Rossi, S. (2014). Efficiency of weak brain connections support general cognitive functioning. Hum. Brain Mapp. 35, 4566-4582. doi:10.1002/hbm.22495

Siegel, M., Donner, T. H., and Engel, A. K. (2012). Spectral fingerprints of large-scale neuronal interactions. Nat. Rev. Neurosci. 13, 121-134. doi:10.1038/nrn3137

Šneidere, K., Mondini, S., and Stepens, A. (2020). Role of EEG in measuring cognitive reserve: A rapid review. Front. Aging Neurosci. 12:249. doi:10.3389/fnagi.2020.00249

Speer, M. E., and Soldan, A. (2015). Cognitive reserve modulates ERPs associated with verbal working memory in healthy younger and older adults. Neurobiol. Aging 36, 1424-1434. doi:10.1016/j.neurobiolaging.2014.12.025

Steffener, J., and Stern, Y. (2012). Exploring the neural basis of cognitive reserve in aging. Biochim. Biophys. Acta 1822, 467-473. doi:10.1016/j.bbadis.2011.09.012

Stern, Y. (2002). What is cognitive reserve? theory and research application of the reserve concept. J. Int. Neuropsychol. Soc. 8, 448-460.

Stern, Y., Arenaza-Urquijo, E. M., Bartrés-Faz, D., Belleville, S., Cantilon, M., Chetelat, G., et al (2020). Whitepaper: Defining and investigating cognitive reserve, brain reserve, and brain maintenance. Alzheimers Dement. 16, 1305-1311. doi:10.1016/j.jalz.2018.07.219

Thompson, M., \& Thompson, L. (2006). Improving attention in adults and children: Differing electroencephalograhy profiles and implications for training. Biofeedback 34, 99-105

Tort, A. B., Hammer, M., Zhang, J., Brankačk, J., \& Draguhn, A. (2021). Temporal relations between cortical network oscillations and breathing frequency during REM sleep. J. Neurosci., 41(24), 5229-5242. doi: 10.1523/JNEUROSCI.3067-20.2021

van Straaten, E. C., de Haan, W., de Waal, H., Scheltens, P., van der Flier, W. M., Barkhof, F., et al. (2012). Disturbed oscillatory brain dynamics in subcortical ischemic vascular dementia. BMC Neurosci. 13:85. doi:10.1186/1471-2202-13-85

Whitham, E. M., Lewis, T., Pope, K. J., Fitzgibbon, S. P., Clark, C. R., Loveless, S., ... \& Willoughby, J. O. (2008). Thinking activates EMG in scalp electrical recordings. Clin. Neurophysiol, 119(5), 1166-1175. doi: 10.1016/j.clinph.2008.01.024

Wook Yoo, S., Han, C. E., Shin, J. S., Won Seo, S., Na, D. L., Kaiser, M. et al. (2015). A network flow-based analysis of cognitive reserve in normal ageing and Alzheimer's disease. Sci. Rep. 5, 1-14. doi: 10.1038/srep10057.

Yang, C. Y., and Lin, C. P. (2020). Classification of cognitive reserve in healthy older adults based on brain activity using support vector machine. Physiol. Meas. 41, 065009. doi: 10.1088/13616579/ab979e.

Yuval-Greenberg, S., \& Deouell, L. Y. (2010). Scalp-recorded induced gamma-band responses to auditory stimulation and its correlations with saccadic muscle-activity. Brain topography, 24(1), 30-39. doi: 10.1007/s10548-010-0157-7 
Yuval-Greenberg, S., Tomer, O., Keren, A. S., Nelken, I., \& Deouell, L. Y. (2008). Transient induced gamma-band response in EEG as a manifestation of miniature saccades. Neuron, 58(3), 429-441. doi: 10.1016/j.neuron.2008.03.027

Zahodne, L. B., Manly, J. J., Brickman, A. M., Narkhede, A., Griffith, E. Y., Guzman, V. A., et al (2015). Is residual memory variance a valid method for quantifying cognitive reserve? A longitudinal application. Neuropsychologia 77, 260-266. doi:

10.1016/j.neuropsychologia.2015.09.009.

Zahodne, L. B., Manly, J. J., Brickman, A. M., Siedlecki, K. L., DeCarli, C., \& Stern, Y. (2013). Quantifying cognitive reserve in older adults by decomposing episodic memory variance: replication and extension. J. Int. Neuropsychol. Soc. 19, 854-862. doi:

$10.1017 / \mathrm{S} 1355617713000738$.

\section{DATA AVAILABILITY STATEMENT}

The dataset analyzed and the results' files can be found online in the Open Science Framework website at the following link: https://osf.io/bdfyu/?view_only=c08b64ac05a948709a8778b608fe716e 


\section{Supplementary Material}

Supplementary Table 1. Pearson's correlation coefficients and $p$-values for the relationship between Age and demographic and cognitive variables $(n=56)$.

\begin{tabular}{|c|c|c|}
\hline & Age & \\
\hline \multirow[t]{2}{*}{ Education years } & $\begin{array}{l}\text { Pearson's } \\
\text { correlation }\end{array}$ & $\begin{array}{r}- \\
0.637\end{array}$ \\
\hline & Sig. (bilateral) & 0.000 \\
\hline \multirow[t]{2}{*}{ CRI Education } & $\begin{array}{l}\text { Pearson's } \\
\text { correlation }\end{array}$ & 0.041 \\
\hline & Sig. (bilateral) & 0.766 \\
\hline \multirow[t]{2}{*}{ CRI Laboural } & $\begin{array}{l}\text { Pearson's } \\
\text { correlation }\end{array}$ & 0.402 \\
\hline & Sig. (bilateral) & 0.002 \\
\hline \multirow[t]{2}{*}{ CRI Leisure } & $\begin{array}{l}\text { Pearson's } \\
\text { correlation }\end{array}$ & 0.305 \\
\hline & Sig. (bilateral) & 0.022 \\
\hline \multirow[t]{2}{*}{ Total CRI } & $\begin{array}{l}\text { Pearson's } \\
\text { correlation }\end{array}$ & 0.235 \\
\hline & Sig. (bilateral) & 0.082 \\
\hline \multirow[t]{2}{*}{ CANTAB RTI } & $\begin{array}{l}\text { Pearson's } \\
\text { correlation }\end{array}$ & 0.347 \\
\hline & Sig. (bilateral) & 0.009 \\
\hline \multirow[t]{2}{*}{ CANTAB PAL } & $\begin{array}{l}\text { Pearson's } \\
\text { correlation }\end{array}$ & 0.703 \\
\hline & Sig. (bilateral) & 0.000 \\
\hline \multirow[t]{2}{*}{ CANTAB SSP } & $\begin{array}{l}\text { Pearson's } \\
\text { correlation }\end{array}$ & 0.638 \\
\hline & Sig. (bilateral) & $\mathbf{0 . 0 0 0}$ \\
\hline \multirow[t]{2}{*}{ CANTAB MMT } & $\begin{array}{l}\text { Pearson's } \\
\text { correlation }\end{array}$ & 0.265 \\
\hline & Sig. (bilateral) & 0.048 \\
\hline \multirow[t]{2}{*}{ CANTAB SWM } & $\begin{array}{l}\text { Pearson's } \\
\text { correlation }\end{array}$ & 0.600 \\
\hline & Sig. (bilateral) & 0.000 \\
\hline \multirow[t]{2}{*}{ CANTAB composite } & $\begin{array}{l}\text { Pearson's } \\
\text { correlation }\end{array}$ & 0.723 \\
\hline & Sig. (bilateral) & 0.000 \\
\hline
\end{tabular}


Supplementary Table 2. Statistics for non-significant moderation models

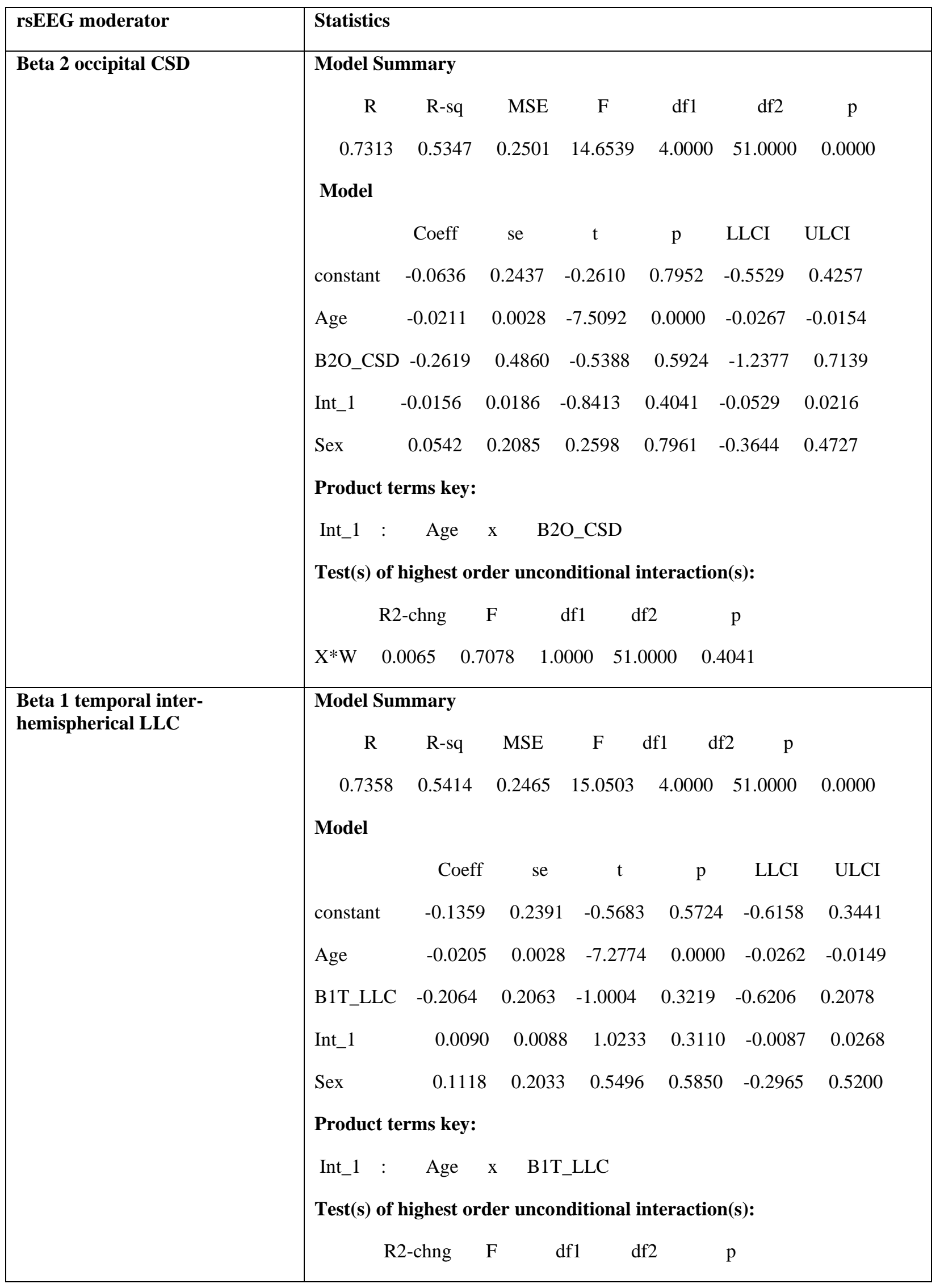




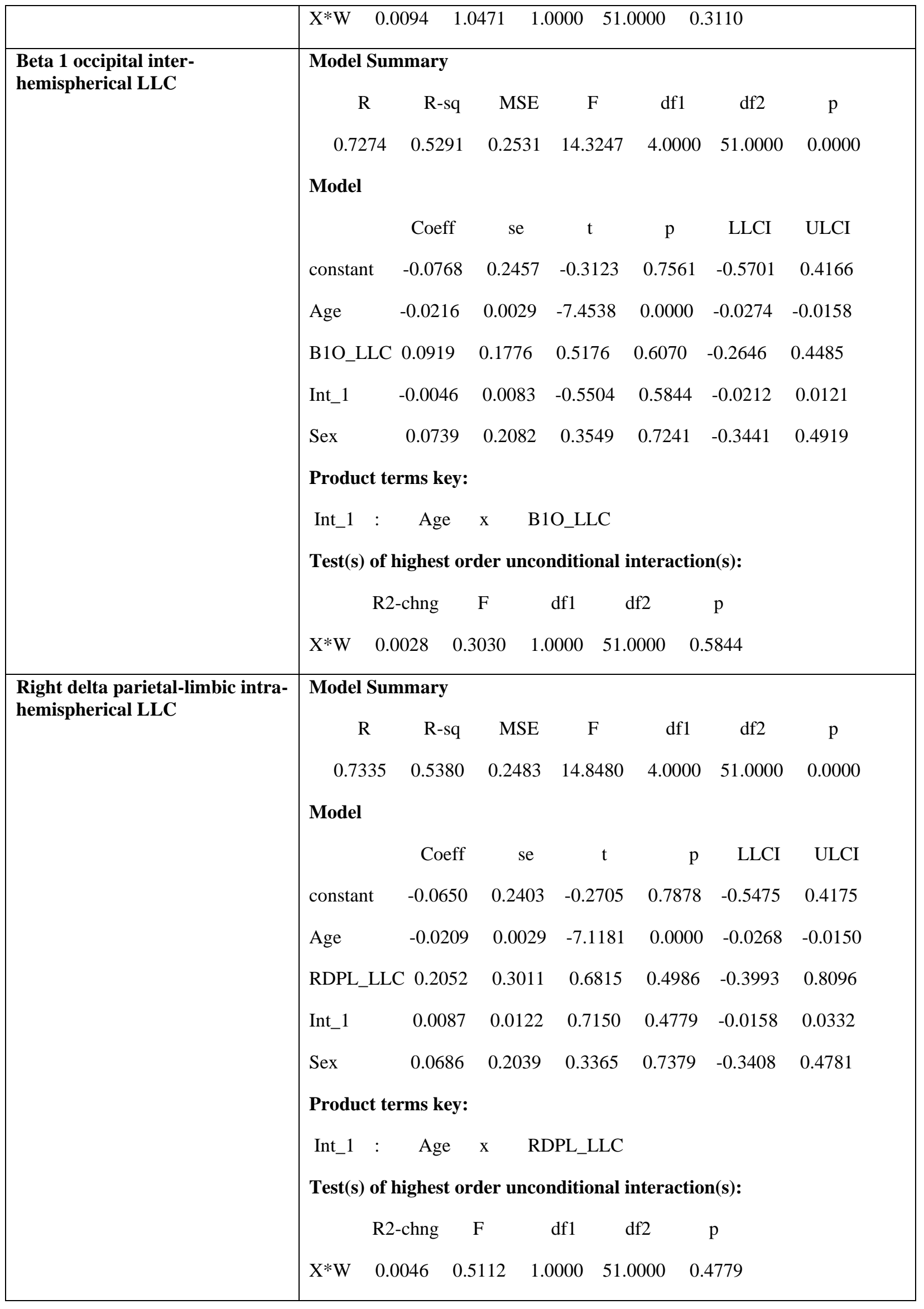




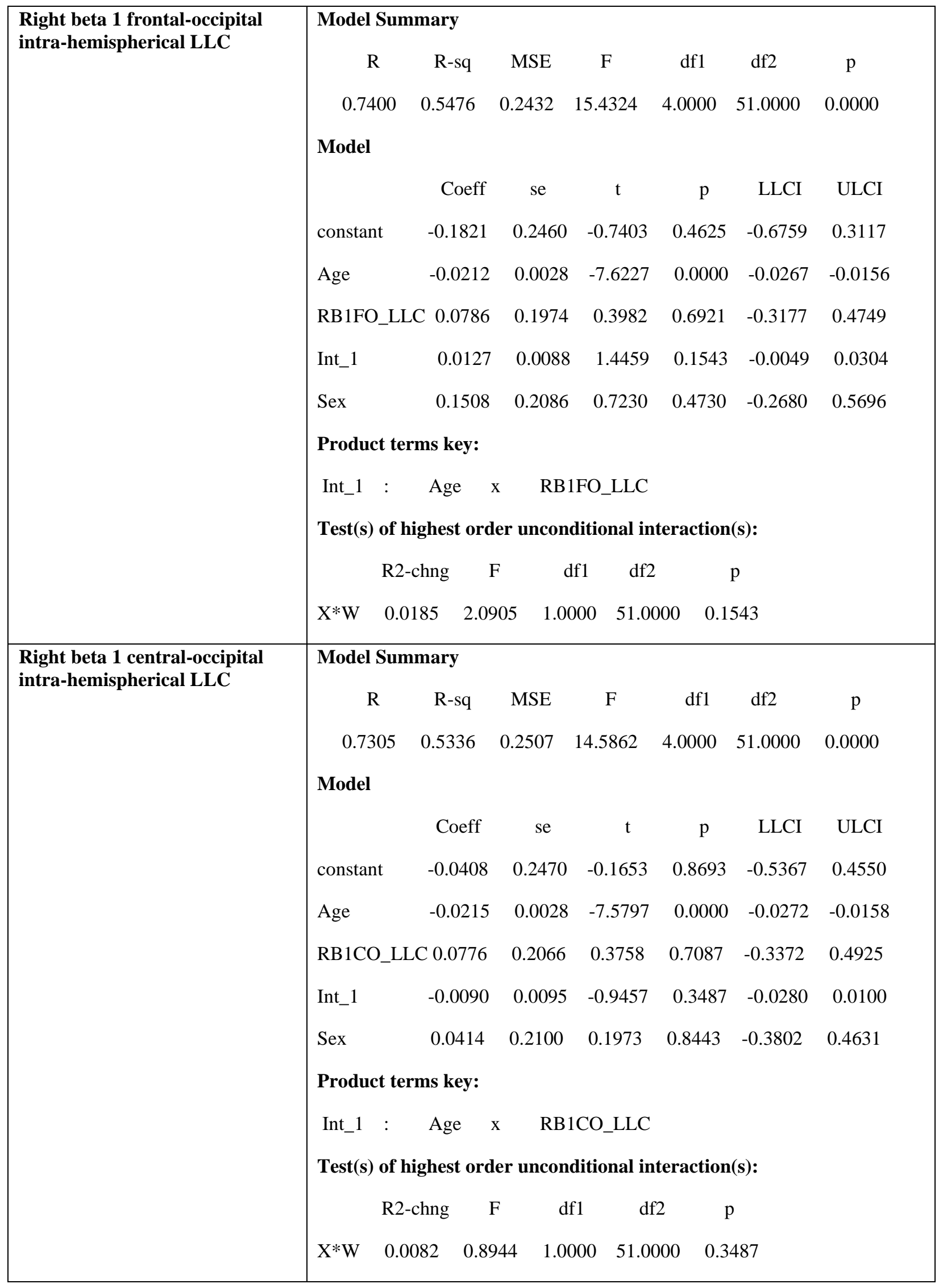




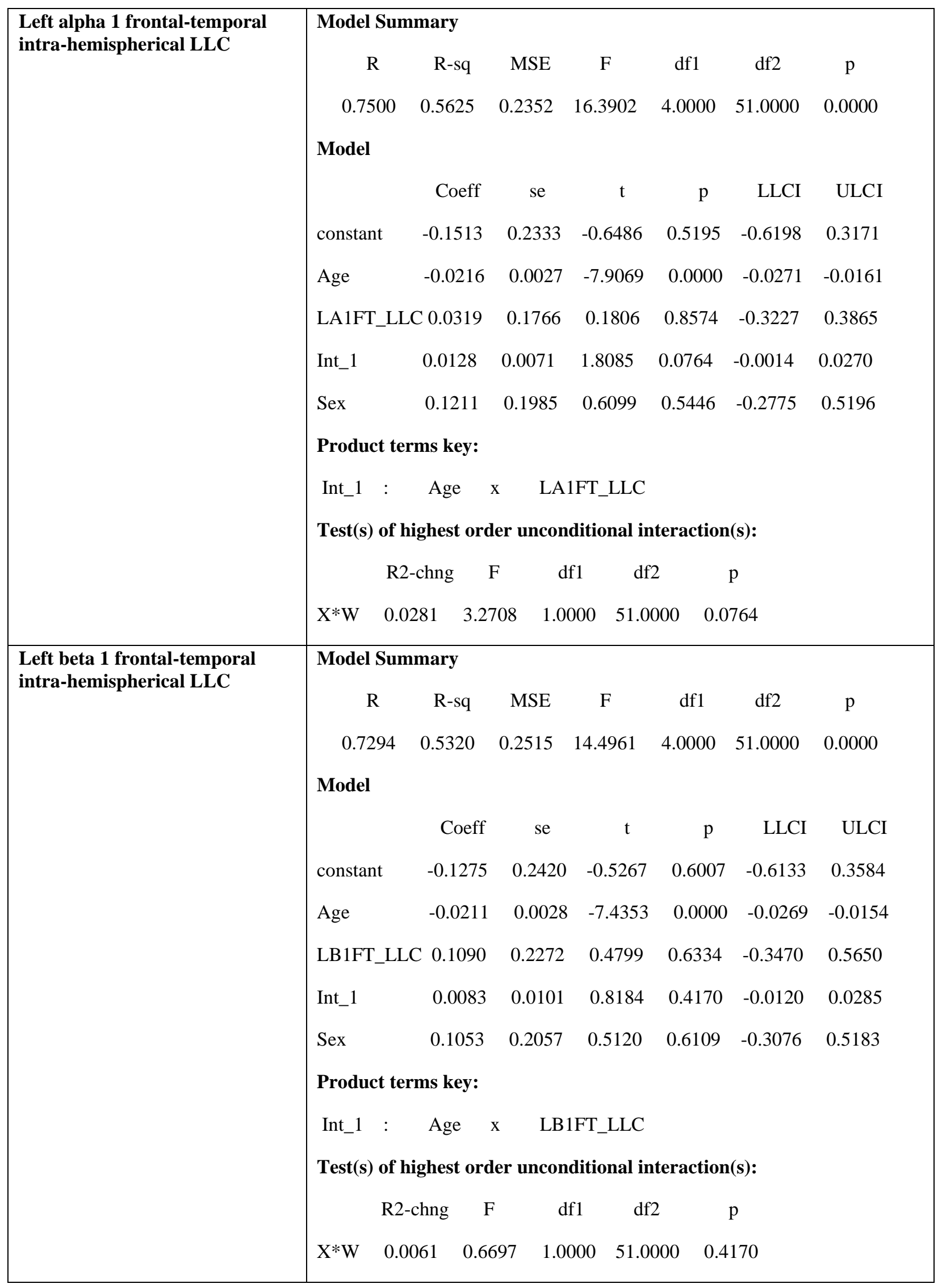




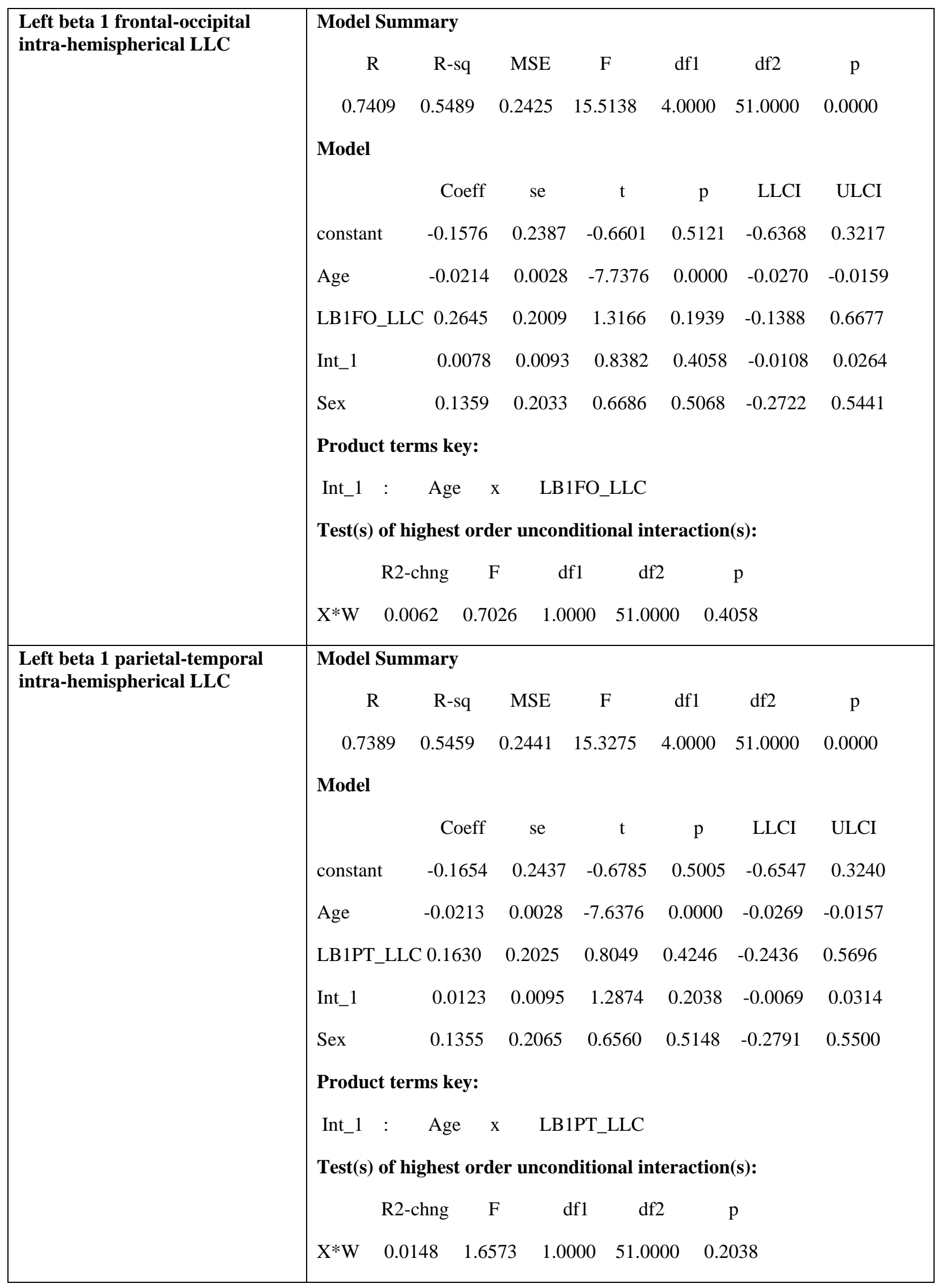




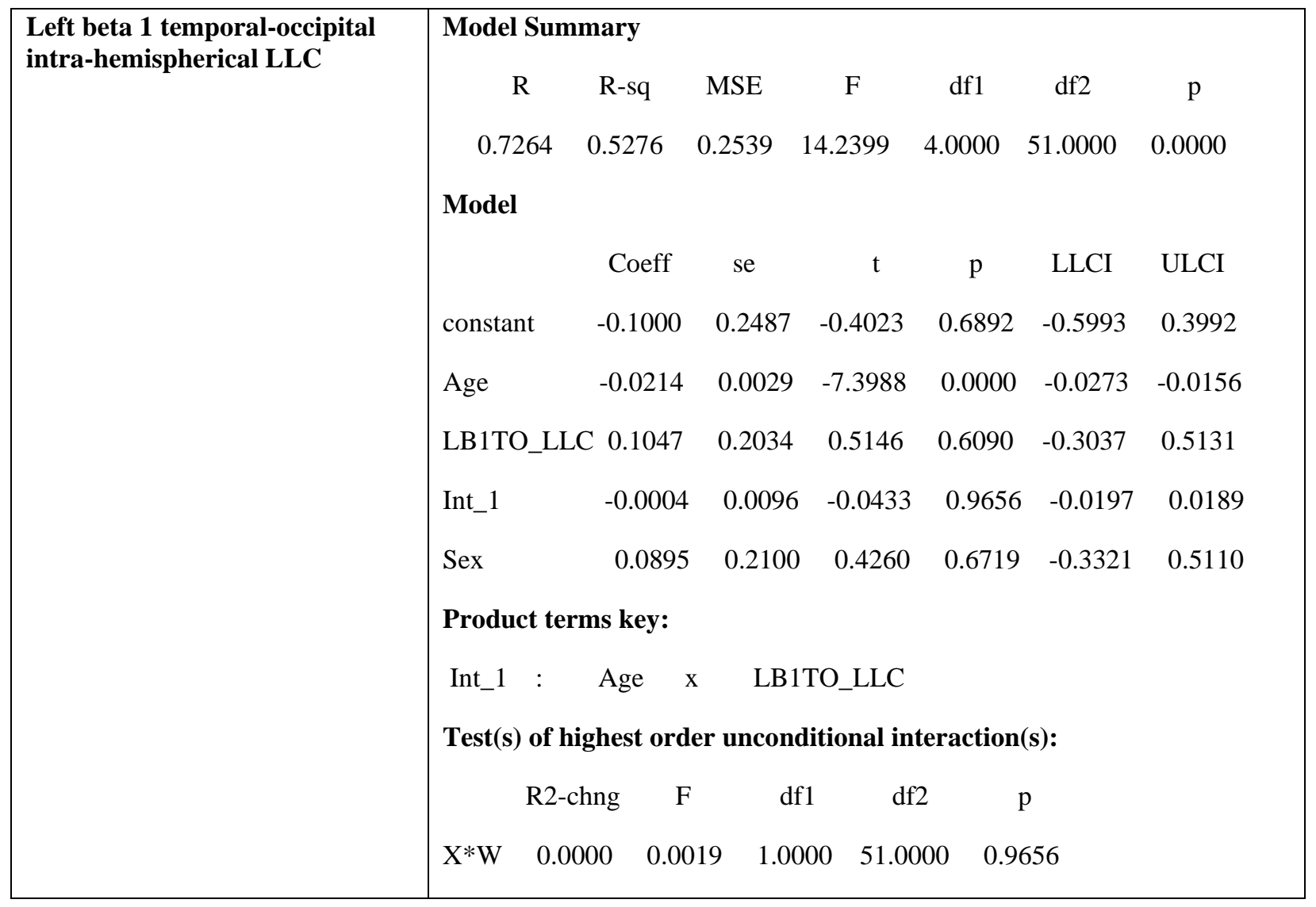

Supplementary Table 3. Statistics for moderation models with CRI measures (total CRI, CRIEducation, CRI-Working Activity and CRI-Leisure) as moderators in the relationship between age and cognitive status.

\begin{tabular}{|c|c|c|c|c|c|c|c|}
\hline CRI Moderator & Statistic & & & & & & \\
\hline Total CRI & Model S & ummary & & & & & \\
\hline & $\mathrm{R}$ & R-sq & MSE & $\mathrm{F}$ & df1 & df2 & $\mathrm{p}$ \\
\hline & 0.734 & 0.5393 & 0.2477 & 14.922 & 4.000 & 51.0000 & 0.0000 \\
\hline & Model & & & & & & \\
\hline & & Coeff & se & $\mathrm{t}$ & $\mathrm{p}$ & LLCI & ULCI \\
\hline & constant & -0.0129 & 0.2512 & -0.0515 & 0.9591 & -0.5173 & 0.4914 \\
\hline & Age & -0.0220 & 0.0030 & -7.2645 & 0.0000 & -0.0281 & -0.0159 \\
\hline & CRI & 0.0012 & 0.0058 & 0.2109 & 0.8338 & -0.0104 & 0.0128 \\
\hline & Int_1 & -0.0003 & 0.0002 & -1.1569 & 0.2527 & -0.0008 & 0.0002 \\
\hline & Sex & 0.0316 & 0.2103 & 0.1505 & 0.8810 & -0.3905 & 0.4537 \\
\hline & Product & terms key & & & & & \\
\hline
\end{tabular}




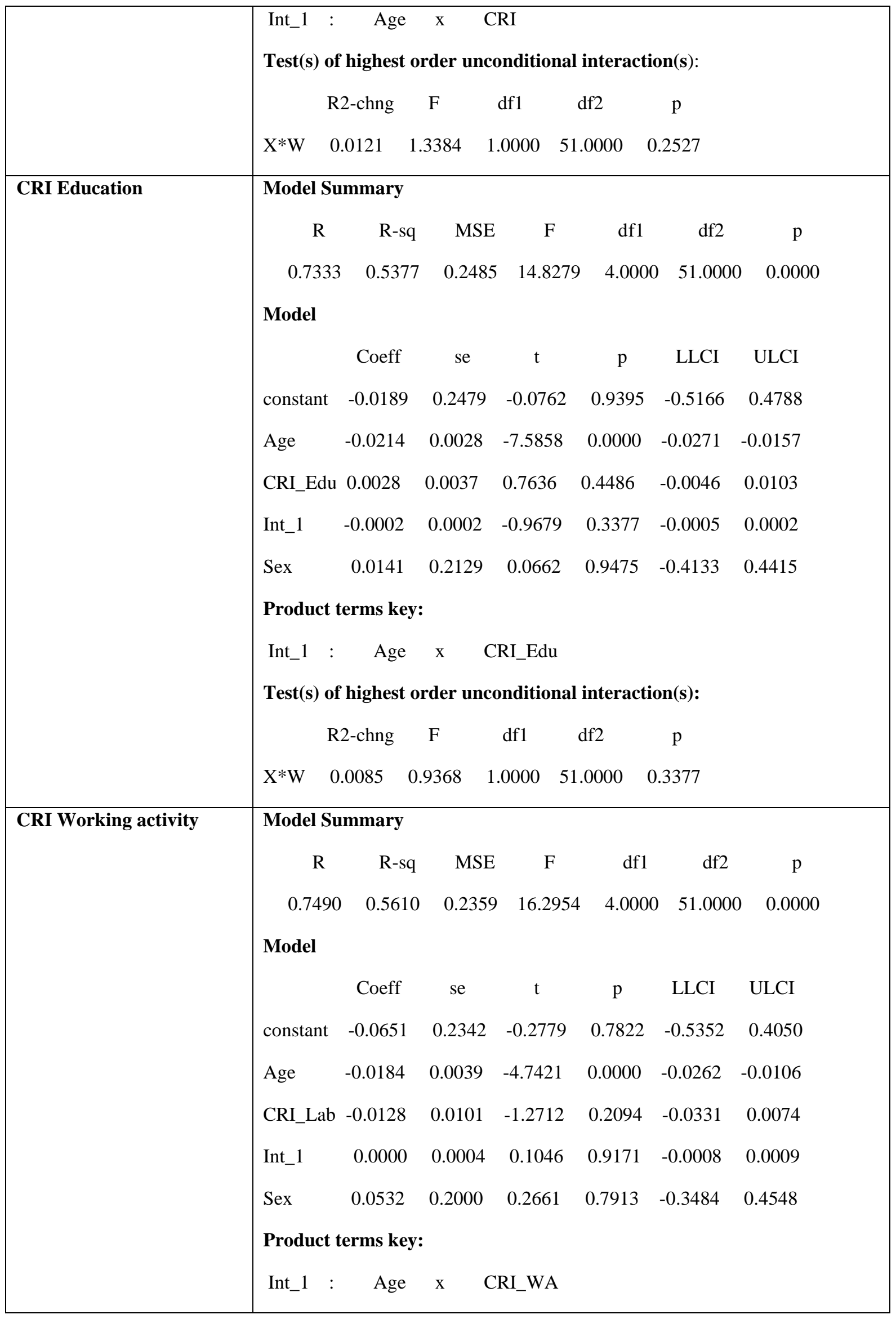




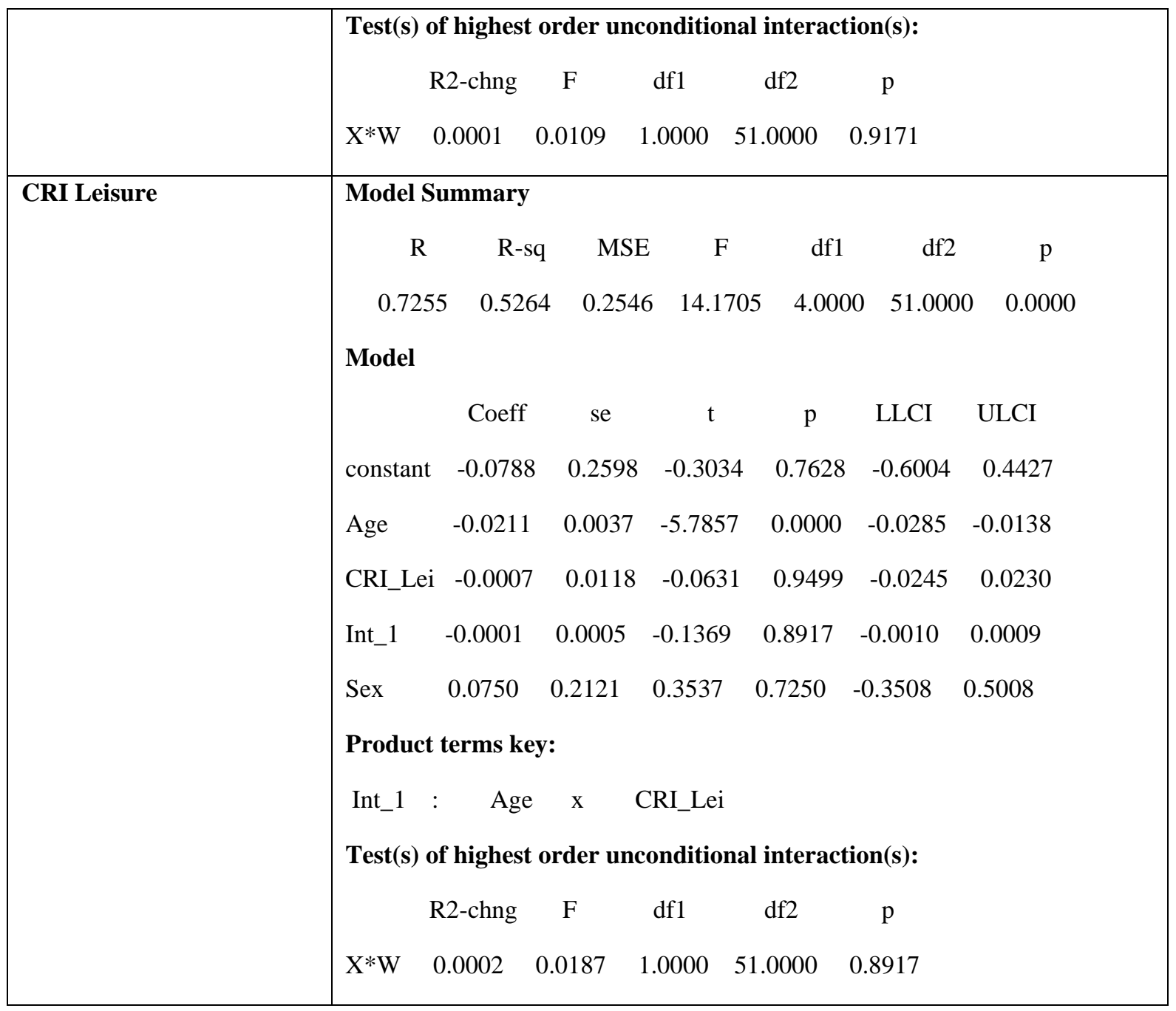

Supplementary Table 4. Statistics for significant interaction terms in moderation analyses using CANTAB sub-scales as dependent variables.

\subsection{CANTAB SSP}

\begin{tabular}{|c|c|c|c|c|c|c|c|}
\hline rsEEG moderator & Statistics & & & & & & \\
\hline \multirow[t]{6}{*}{ Delta occipital CSD } & \multicolumn{7}{|c|}{ Model Summary } \\
\hline & $\mathrm{R}$ & $\mathrm{R}$-sq & MSE & $\mathrm{F}$ & df1 & df 2 & $\mathrm{p}$ \\
\hline & 0.6958 & 0.4841 & 1.5388 & 11.9643 & 4.0000 & 51.0000 & 0.0000 \\
\hline & \multicolumn{7}{|l|}{ Model } \\
\hline & & Coeff & se & $\mathrm{t}$ & $\mathrm{p}$ & LLCI & ULCI \\
\hline & constant & 4.7568 & 0.6116 & 7.7777 & 0.0000 & 3.5290 & 5.9846 \\
\hline
\end{tabular}




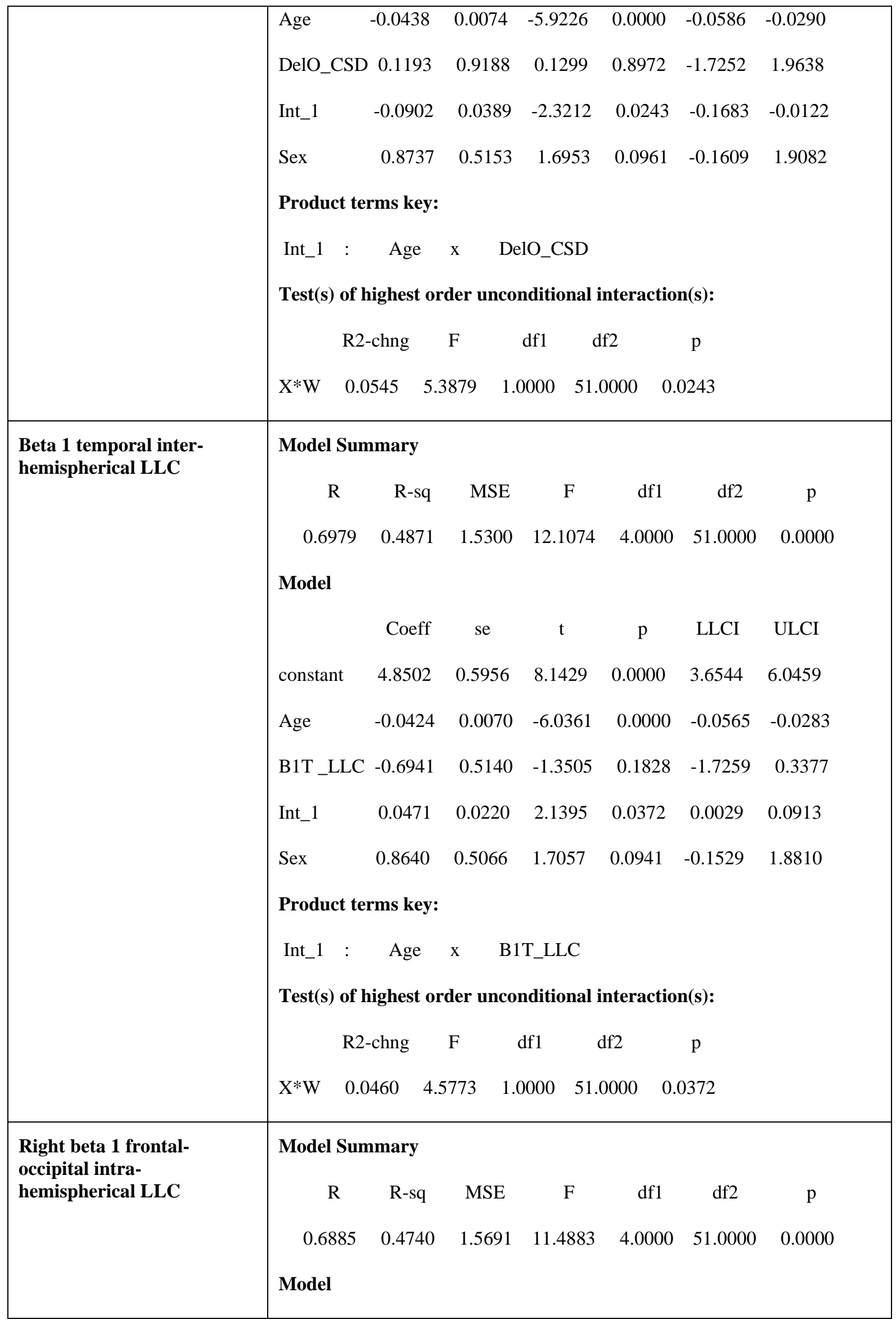




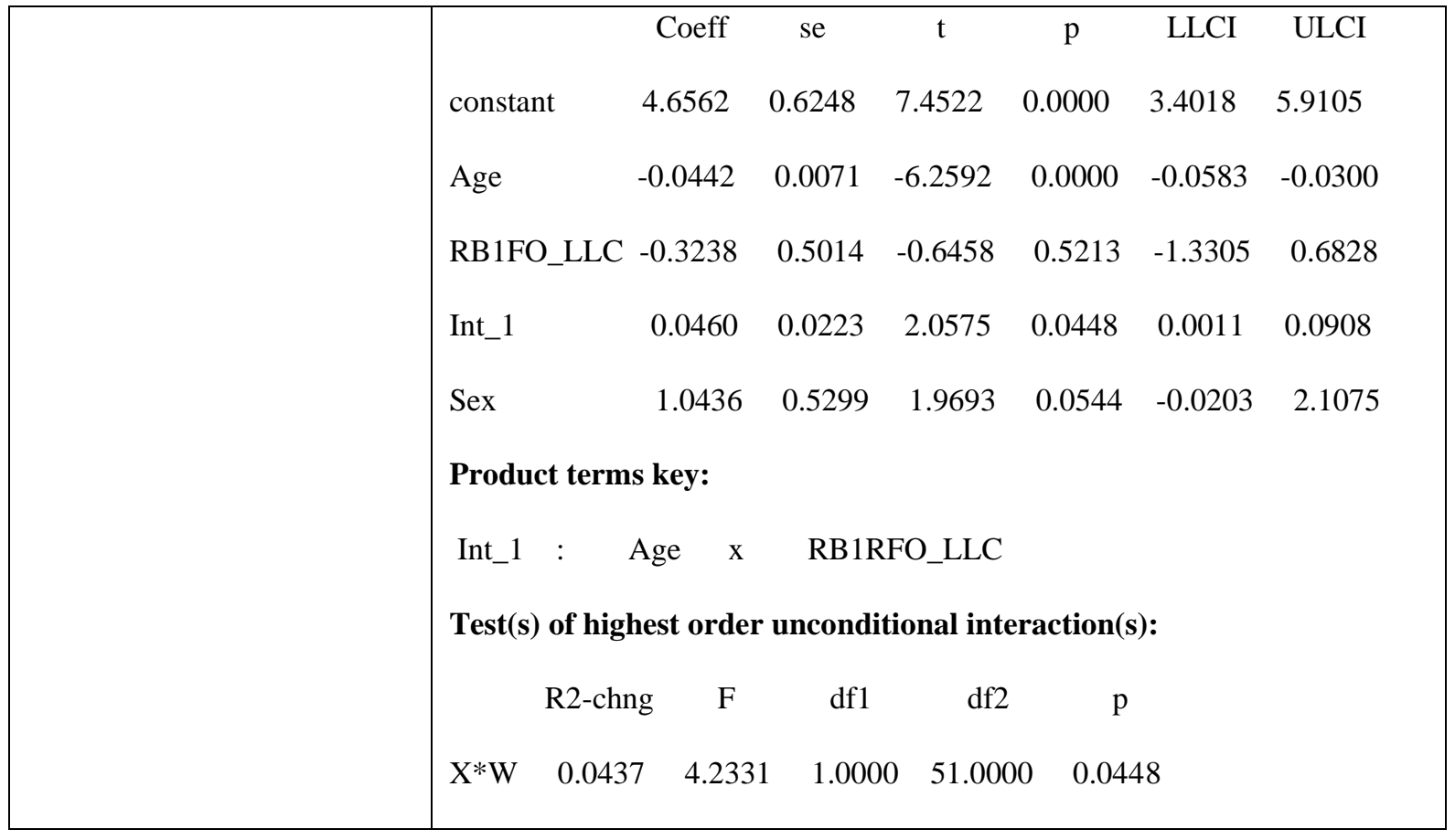

\subsection{CANTAB SWM}

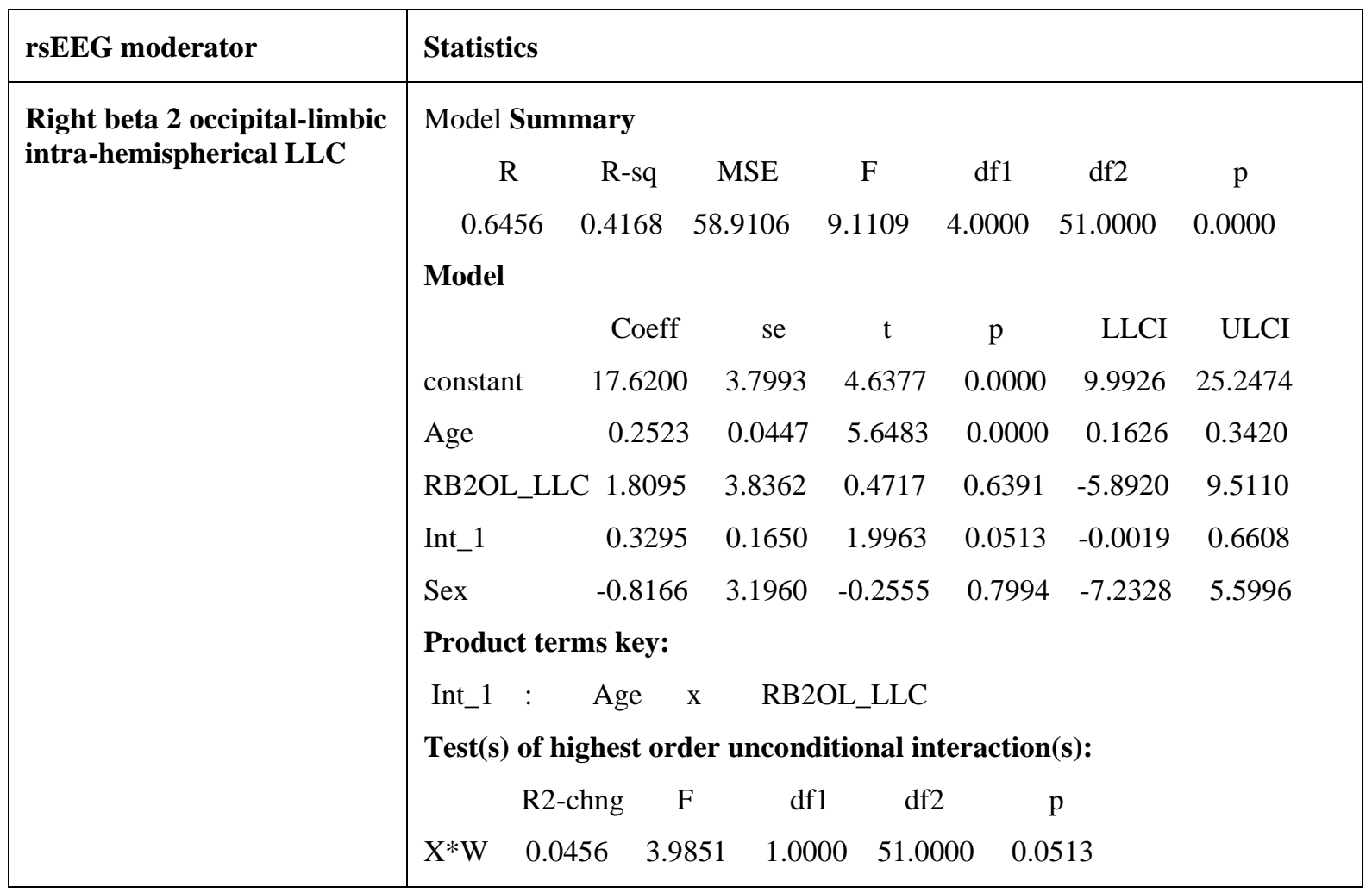

\section{Supplementary information:}




\section{1) Gamma analysis and results}

Given the exploratory nature of the present study, we have performed gamma analysis in order to cover the whole frequencies spectra. The procedure was exactly the same as for the rest of frequency bands. The cortical sources of spectral density with a resolution of $0.5 \mathrm{~Hz}$ were estimated for a range between 30.5 to $40 \mathrm{~Hz}$. Also, the lagged-linear connectivity (LLC) was calculated as a measure of interdependence of rsEEG sources.

Gamma rsEEG variables were transformed to a logarithmic scale (log-10). No outliers were detected. The association between the total CRI and the gamma rsEEG variables was tested through a series of bivariate correlations (6 CSD variables; 6 inter-hemispheric LLC variables; and 30 intra-hemispheric LLC variables). Finally, a moderation analysis using the regression-based approach in Hayes' (2017) PROCESS macro (Version 3.5) for IBM SPSS was conducted (Model 1; see Figure 2), with Age as a continuous independent variable (X), CANTAB composite score as the dependent variable (Y), and the gamma rsEEG variables with a moderate correlation with the total CRI as moderator variables. Sex was included as a covariate.

Just one gamma variable showed a moderate effect size correlation with total CRI: the intrahemispheric LLC involving parietal and temporal regions in the right hemisphere (Pearson's correlation $=0.32 ; 95 \% \mathrm{CI}=0.07-0.51)$. However, this variable did not moderate the relationship between age and cognitive status, being the interaction term between age and intra-hemispheric LLC between parietal and temporal regions for gamma activity in the right hemisphere not significant, $b=-0.004$, $t(51)=-0.38$, BootLLCI $=-0.023$-BootULCI $=0.016 ; p=0.71 ; \Delta R^{2}=0.001, F(1,51)=0.144$.

This moderate correlation is partially in agreement with previous gamma results related to cognitive reserve. For example, Fleck et al. (2017) found greater connectivity for gamma band in high-CR than in low-CR participants but just for older adults, not for the younger participants in their study. Similarly, Yang and Lin (2020) found higher resting-state gamma intensity in the right temporal region for participants with high than low CR, in a MEG study. These authors interpreted their findings as evidence for better neurocognitive activities such as perception and working memory in healthy aging (Yang \& Lin, 2020). Moreover, the higher levels of gamma activity in the right hemisphere for older adults were interpreted as a mechanism for neural compensation in high-CR (Fleck et al., 2017).

\section{2) Multiple Imputation procedure}


The APA Task Force on Statistical Inference explicitly warned against the use of simple methods of imputation such as listwise or pairwise deletion (see Dong \& Peng, 2013) when dealing with missing values. For multiple imputation procedures, instead of filling in a single value for each missing value, a set of plausible values that represent the uncertainty about the right value to impute are produced for each missing value. This process results in valid statistical inferences that properly reflect the uncertainty due to missing values (Yuan, 2000). Predictive mean matching (PMM), the specific multiple imputation procedure used in the current study is now one of the most accepted methods for multiple imputation among researchers in social sciences due to its robustness and bias-free estimates. It is a hot deck procedure, which finds a suitable "donor" for a missing case by using similar (in some characteristics) observed cases. Thus, drawing the "donor" case from a pool. To do so, it matches the cases via the closeness of the predicted means (in a regression model). The "winner donor" in the pool is the one with predicted values closer to the predicted values for the missing case. We chose this procedure since it is less sensitive than parametric procedures to regression model misspecifications, such as nonlinear associations, heteroscedasticity, deviations from normality or when the assumptions for the regression model are unmet (Morris, White, \& Royston, 2014). That way, PMM imputed data usually preserves the distribution of the observed data (see below). Furthermore, PMM imputations yield "valid" values, in the sense they will fit the respective scale of measurement for a given variable, and will always lie within the range of observed values. Additionally, a recent simulation study has shown it is a robust and bias-free procedure in samples as small as $\mathrm{N}=20$ even if combined with large amounts of missing data (40 - $50 \%$ ) (Kleinke, 2018).

So, given the observed percentage of missing data was within the range of those commonly encountered (15-20\%) in educational and psychological studies (Enders, 2003), we decided to proceed with the multiple imputation.

First, in order to check that the missing data do not follow a defined pattern, which is a requisite for multiple imputation of missing data, we performed the Missing Completely at Random (MCAR) test of Little (Little, 1988). Little's MCAR test results were non-significant; thus, we can assure that the missing values were completely at random. Further, to check that the multiple imputation process had worked properly, we compared the distribution of the variables from the original sample without imputations with the distribution of the same variables after the multiple imputation process. To that end, we conducted a one-way ANOVA, which pointed to the absence of significant mean differences between the two conditions for any of the CANTAB subscales: 
- RTI (56 participants versus 45): means 298.12 versus 296.99; $F(1)=0.005, p=0.95$

- PAL (56 participants versus 45): means 17.13 versus 17.16; $F(1)=0.000, p=0.99$

- $\mathrm{SWM}$ (56 participants versus 45): means 16.11 versus $16.11 ; \mathrm{F}(1)=0.000, \mathrm{p}=0.99$

- SSP (56 participants versus 44): means 5.88 versus $5.86 ; \mathrm{F}(1)=0.001, \mathrm{p}=0.98$

- MMT (56 participants versus 44): means 254.88 versus $254.85, F(1)=0.000, p=0.99$

\section{SUPPLEMENTARY REFERENCES}

Dong, Y., \& Peng, C. Y. J. (2013). Principled missing data methods for researchers. SpringerPlus, 2(1), 1-17

Enders, C. K. (2003). Using the Expectation Maximization Algorithm to Estimate Coefficient Alpha for Scales With Item-Level Missing Data. Psychol. Meth., 8(3), 322-337. doi: 10.1037/1082989X.8.3.322

Fleck, J. I., Kuti, J., Mercurio, J., Mullen, S., Austin, K., and Pereira, O. (2017). The impact of age and cognitive reserve on resting-state brain connectivity. Front. Aging Neurosci. $9: 392$. doi:10.3389/fnagi.2017.00392

Hayes, A. F. (2017). Introduction to mediation, moderation, and conditional process analysis: A regression-based approach. New York: Guilford Press.

Kleinke, K. (2018). Multiple imputation by predictive mean matching when sample size is small. Methodology 14, 3-15

Little, R. J. (1988). A test of missing completely at random for multivariate data with missing values. Journal of the American statistical Association, 83(404), 1198-1202.).

Morris, T. P., White, I. R and Royston, P. (2014). Tuning multiple imputation by predictive mean matching and local residual draws. BMC medical research methodology 14.1, 1-13

Yang, C. Y., and Lin, C. P. (2020). Classification of cognitive reserve in healthy older adults based on brain activity using support vector machine. Physiol. Meas. 41, 065009. doi: 10.1088/13616579/ab979e.

Yuan, Y. C. (2000). Multiple imputation for missing data: Concepts and new development. In Proceedings of the Twenty-Fifth Annual SAS Users Group International Conference (Vol. 267, No. 11 